\title{
SMALL GAIN THEOREM WITH RESTRICTIONS FOR UNCERTAIN TIME-VARYING NONLINEAR SYSTEMS
}

\author{
MINGHUI ZHU* AND JIE HUANG ${ }^{\dagger}$
}

\begin{abstract}
This paper gives three versions of the small gain theorem with restrictions for uncertain time-varying nonlinear systems. The result can be viewed as an extension of the small gain theorem with restrictions for time-invariant nonlinear systems or the small gain theorem without restrictions for time-varying nonlinear systems. The result can be applied to study the stabilization problem or the output regulation problem of uncertain nonlinear systems.
\end{abstract}

Index Terms - small gain theorem, nonlinear control, nonlinear systems.

The small gain theorem is an important tool to ascertain the stability of two interconnected systems assuming each of the individual systems is stable in some sense. Small gain theorem has many different versions under various stability concepts [2] to [14], [20]. In this paper, we will focus on the small gain theorem in the context of inputto-state and/or input-to-output stability [15] to [19]. The first small gain theorem for nonlinear time-varying systems in the input-to-state stability (ISS) framework was established by Jiang et al [7]. The resulting small gain condition is given in terms of two inequalities. Recently, Chen and Huang further considered the small gain theorem for uncertain time-varying nonlinear system [2]. They presented a simplified small gain condition which is in a familiar form of the contraction mapping known for time-invariant nonlinear systems [7].

In [20], Teel introduced the concept of ISS with restrictions on the initial states and inputs and established a small gain theorem with restrictions for time-invariant systems. In Appendix B of [6], relying upon the separation property for ISS with restrictions, Isidori et al established a more general small gain theorem with restrictions for time-invariant systems. Nevertheless, the proof of [6] cannot be carried over to the case of time-varying systems, because the separation property for ISS does not hold for time-varying systems [2].

This paper is to establish three versions of the small gain theorem with restrictions for uncertain time-varying nonlinear systems, thus filling the gap between the small gain theorem with restrictions for time-varying nonlinear systems and that for time invariant nonlinear systems.

\footnotetext{
* Department of Mechanical and Automation Engineering, The Chinese University of Hong Kong, Shatin, N.T., Hong Kong, E-mail: mhzhu@mae.cuhk.edu.hk

${ }^{\dagger}$ Corresponding author: Jie Huang, Chinese University of Hong Kong. The work described in this paper was partially supported by the Hong Kong Research Grant Council under CUHK 412006. E-mail: jhuang@mae.cuhk.edu.hk
} 
1. Preliminary. Throughout the paper, let $L_{\infty}^{m}$ be the set of all piecewise continuous bounded functions $u:\left[t_{0}, \infty\right) \mapsto \Re^{m}$ with a finite supremum norm $\left\|u_{\left[t_{0}, \infty\right)}\right\|=$ $\sup _{t \geq t_{0}}\|u(t)\|$. Denote the supremum norm of the truncation of $u(t)$ in $\left[t_{1}, t_{2}\right]$ by $\left\|u_{\left[t_{1}, t_{2}\right]}\right\|=\sup _{t_{1} \leq t \leq t_{2}}\|u(t)\|$. And denote $\|u\|_{a}=\limsup _{t \rightarrow \infty}\|u\|$. A continuous function $\gamma: \Re_{\geq 0} \mapsto \Re_{\geq 0}$ is of class $K$ if it is strictly increasing and $\gamma(0)=0$; and a continuous function $\beta(s, t): \Re_{\geq 0} \times \Re_{\geq 0} \mapsto \Re_{\geq 0}$ is of class $K L$ if, for each fixed $t \geq 0$, the function $\beta(s, t)$ belongs to class $K$ with respect to $s$ and, for each fixed $s$, the function $\beta(s, t)$ is decreasing with respect to $t$, and $\beta(s, t) \rightarrow 0$ as $t \rightarrow \infty$.

Consider the following time-varying uncertain nonlinear system

$$
\begin{aligned}
\dot{x} & =f(x, u, d, t), \\
y & =h(x, u, d, t) \quad t \geq t_{0} \geq 0
\end{aligned}
$$

where $x \in \Re^{n}$ is the plant state, $u \in \Re^{m}$ the input, $y \in \Re^{p}$ the output, $t_{0}$ the initial time, the functions $f: \Re^{n} \times \Re^{m} \times \Re^{n_{d}} \times\left[t_{0}, \infty\right) \mapsto \Re^{n}$ and $h: \Re^{n} \times \Re^{m} \times \Re^{n_{d}} \times$ $\left[t_{0}, \infty\right) \mapsto \Re^{p}$ are piecewise continuous in $t$ and locally Lipschitz in $\operatorname{col}(x, u, d)$. And $d(t):\left[t_{0}, \infty\right) \mapsto \Re^{n_{d}}$ is a family of piecewise continuous functions of $t$, representing the external disturbance and/or the internal uncertainty.

Definition 1.1. System (1) is said to be (uniformly) robust input-to-state stable (RISS) with restrictions $X \subset \Re^{n}$ and $\Delta>0$ on the initial state $x\left(t_{0}\right)$ and the input $u$ respectively if there exist class $K L$ function $\beta$ and class $K$ function $\gamma$, independent of $d(t)$, such that, for any initial state $x\left(t_{0}\right) \in X$ and any input function $u(t) \in L_{\infty}^{m}$ satisfying $\left\|u_{\left[t_{0}, \infty\right)}\right\|<\Delta$, the solution of (1) exists and satisfies, for all $t \geq t_{0}$,

$$
\|x(t)\| \leq \max \left\{\beta\left(\left\|x\left(t_{0}\right)\right\|, t-t_{0}\right), \gamma\left(\left\|u_{\left[t_{0}, t\right]}\right\|\right)\right\} .
$$

Definition 1.2. System (1) is said to be robust input-to-output stable (RIOS) with restrictions $X$ and $\Delta$ on the initial state $x\left(t_{0}\right)$ and the input $u$ respectively if there exist class $K L$ function $\beta$ and class $K$ function $\gamma$, independent of $d(t)$, such that, for any initial state $x\left(t_{0}\right) \in X$, any input function $u(t) \in L_{\infty}^{m}$ satisfying $\left\|u_{\left[t_{0}, \infty\right)}\right\|<\Delta$, the output of (1) exists and satisfies, for all $t \geq t_{0}$,

$$
\|y(t)\| \leq \max \left\{\beta\left(\left\|x\left(t_{0}\right)\right\|, t-t_{0}\right), \gamma\left(\left\|u_{\left[t_{0}, t\right]}\right\|\right)\right\} .
$$

Definition 1.3. System (1) is said to be semi-uniformly RISS with restrictions $X$ and $\Delta$ on the initial state $x\left(t_{0}\right)$ and the input $u$ respectively if there exist class $K$ functions $\gamma^{0}$ and $\gamma^{u}$, independent of $d(t)$, such that for any initial state $x\left(t_{0}\right) \in X$ 
and input $u \in L_{\infty}^{m}$ satisfying $\left\|u_{\left[t_{0}, \infty\right)}\right\|<\Delta$, the solution of (1) exists and satisfies, for all $t \geq t_{0}$,

$$
\begin{aligned}
\|x(t)\| & \leq \max \left\{\gamma^{0}\left(\left\|x\left(t_{0}\right)\right\|\right), \gamma^{u}\left(\left\|u_{\left[t_{0}, \infty\right)}\right\|\right)\right\} \\
\|x\|_{a} & \leq \gamma^{u}\left(\|u\|_{a}\right) .
\end{aligned}
$$

REMARK 1.1. In [17], it was shown that, for the class of time-invariant systems, ISS is equivalent to semi-uniformly ISS. Such equivalence is called separation property. This equivalent relation can also be extended to ISS with restrictions and semi-uniformly ISS with restrictions (Appendix B of [6]). Unfortunately, the separation property does not hold for the time-varying nonlinear systems [2].

Definition 1.4. System (1) is said to satisfy robust asymptotic gain (RAG) property with restrictions $X$ and $\Delta$ on the initial state $x\left(t_{0}\right)$ and the input $u$ respectively if there exists a class $K$ function $\gamma^{u}$, independent of $d(t)$, such that for any initial state $x\left(t_{0}\right) \in X$ and input $u \in L_{\infty}^{m}$ satisfying $\|u\|_{a} \leq \Delta$, the solution of (1) exists and satisfies, for all $t \geq t_{0}$,

$$
\|x\|_{a} \leq \gamma^{u}\left(\|u\|_{a}\right) .
$$

DEFINITION 1.5. The output function of (1) is said to satisfy robust asymptotic $L_{\infty}$ stability (RALS) with restrictions $X$ and $\Delta$ on the initial state $x\left(t_{0}\right)$ and the input $u$ respectively if there exist class $K$ functions $\gamma^{0}$ and $\gamma^{u}$, independent of $d(t)$, such that for any initial state $x\left(t_{0}\right) \in X$ and input $u \in L_{\infty}^{m}$ satisfying $\left\|u_{\left[t_{0}, \infty\right)}\right\|<\Delta$, the output of (1) exists and satisfies, for all $t \geq t_{0}$,

$$
\begin{aligned}
\|y(t)\| & \leq \max \left\{\gamma^{0}\left(\left\|x\left(t_{0}\right)\right\|\right), \gamma^{u}\left(\left\|u_{\left[t_{0}, \infty\right)}\right\|\right)\right\} \\
\|y\|_{a} & \leq \gamma^{u}\left(\|u\|_{a}\right) .
\end{aligned}
$$

Definition 1.6. System(1) is said to satisfy output robust asymptotic gain (o-RAG) property with restrictions $X$ and $\Delta$ on the initial state $x\left(t_{0}\right)$ and the input $u$ respectively if there exists class $K$ function $\gamma^{u}$, independent of $d(t)$, such that for any initial state $x\left(t_{0}\right) \in X$ and input $u \in L_{\infty}^{m}$ satisfying $\|u\|_{a} \leq \Delta$, the output of (1) exists and satisfies, for all $t \geq t_{0}$,

$$
\|y\|_{a} \leq \gamma^{u}\left(\|u\|_{a}\right)
$$




\section{Small Gain Theorem with Restrictions for Uncertain Nonlinear}

\section{Time-varying Systems.}

2.1. The Case of Time Invariant Nonlinear Systems. Consider the feedback interconnection as depicted in Figure 1,

$$
\begin{array}{ll}
\dot{x}_{1}=f_{1}\left(x_{1}, v_{1}, u_{1}\right), & y_{1}=h_{1}\left(x_{1}, v_{1}, u_{1}\right) \\
\dot{x}_{2}=f_{2}\left(x_{2}, v_{2}, u_{2}\right), & y_{2}=h_{2}\left(x_{2}, v_{2}, u_{2}\right)
\end{array}
$$

subject to the following interconnection:

$$
v_{1}=y_{2}, \quad v_{2}=y_{1}
$$

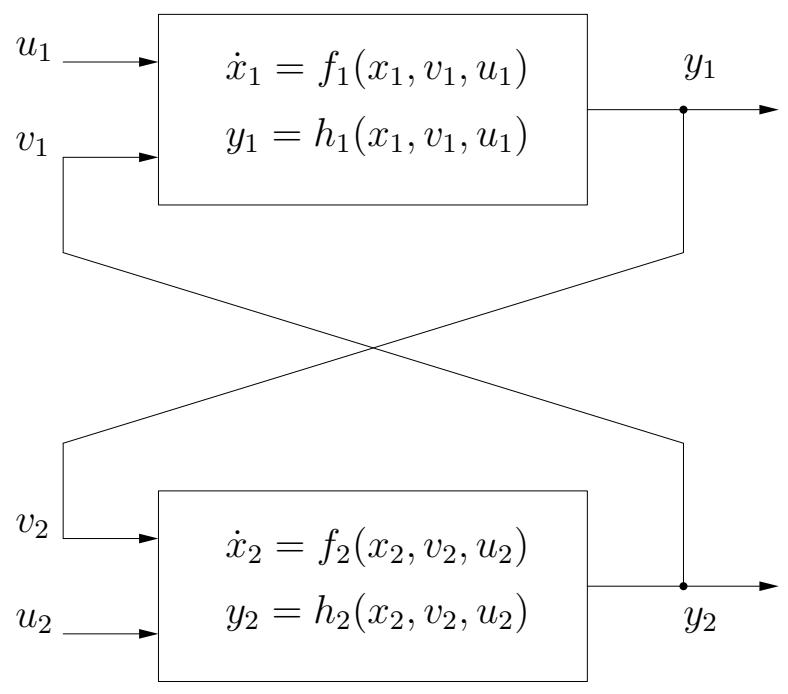

FIG. 1. Inter-connection of (6) and (7)

where, for $i=1,2, x_{i} \in \Re^{n_{i}}, u_{i} \in \Re^{m_{i}}, y_{i} \in \Re^{p_{i}}, v_{i} \in \Re^{q_{i}}$ with $p_{1}=q_{2}, p_{2}=q_{1}$, the function $f_{i}\left(x_{i}, v_{i}, u_{i}\right)$ is locally Lipschitz in $\operatorname{col}\left(x_{i}, v_{i}, u_{i}\right)$, and $f_{i}(0,0,0)=0$, $h_{i}(0,0,0)=0$.

And suppose the following assumption holds.

Assumption 2.1. There exists a $C^{1}$ function $h$ such that

$$
\operatorname{col}\left(y_{1}, y_{2}\right)=h\left(x_{1}, x_{2}, u_{1}, u_{2}\right)
$$

is the unique solution of the equations

$$
y_{1}=h_{1}\left(x_{1}, y_{2}, u_{1}\right), \quad y_{2}=h_{2}\left(x_{2}, y_{1}, u_{2}\right) .
$$


The following small gain theorem with restrictions for time invariant nonlinear systems was established in Appendix B of [6].

Theorem 2.1. Assume that subsystem (6) is ISS with restrictions $X_{1}, \Delta_{1}$ and $\Delta_{1}^{u}$ on $x_{1}(0), v_{1}$ and $u_{1}$ respectively and subsystem (7) is ISS with restrictions $X_{2}$, $\Delta_{2}$ and $\Delta_{2}^{u}$ on $x_{2}(0), v_{2}$ and $u_{2}$ respectively, i.e., there exist class $K L$ functions $\beta_{1}$ and $\beta_{2}$, class $K$ functions $\gamma_{1}, \gamma_{2}, \gamma_{1}^{u}, \gamma_{2}^{u}$ such that, for any $x_{1}(0) \in X_{1}, v_{1}(t) \in L_{\infty}^{q_{1}}$ satisfying $\left\|v_{1[0, \infty)}\right\|<\Delta_{1}, u_{1}(t) \in L_{\infty}^{m_{1}}$ satisfying $\left\|u_{1[0, \infty)}\right\|<\Delta_{1}^{u}$, the solution of $(6)$ exists and satisfies, for all $t \geq 0$,

$$
\left\|x_{1}(t)\right\| \leq \max \left\{\beta_{1}\left(\left\|x_{1}(0)\right\|, t\right), \gamma_{1}\left(\left\|v_{1[0, \infty)}\right\|\right), \gamma_{1}^{u}\left(\left\|u_{1[0, \infty)}\right\|\right)\right\}
$$

and for any $x_{2}(0) \in X_{2}, v_{2}(t) \in L_{\infty}^{q_{2}}$ satisfying $\left\|v_{2[0, \infty)}\right\|<\Delta_{2}, u_{2}(t) \in L_{\infty}^{m_{2}}$ satisfying $\left\|u_{2[0, \infty)}\right\|<\Delta_{2}^{u}$, the solution of (7) exists and satisfies, for all $t \geq 0$,

$$
\left\|x_{2}(t)\right\| \leq \max \left\{\beta_{2}\left(\left\|x_{2}(0)\right\|, t\right), \gamma_{2}\left(\left\|v_{2[0, \infty)}\right\|\right), \gamma_{2}^{u}\left(\left\|u_{2[0, \infty)}\right\|\right)\right\} .
$$

Suppose the following estimates hold for the outputs $y_{1}$ and $y_{2}$

$$
\begin{aligned}
\left\|y_{1[0, \infty)}\right\| & \leq \max \left\{\bar{\gamma}_{1}^{0}\left(\left\|x_{1}(0)\right\|\right), \bar{\gamma}_{1}\left(\left\|v_{1[0, \infty)}\right\|\right), \bar{\gamma}_{1}^{u}\left(\left\|u_{1[0, \infty)}\right\|\right)\right\} \\
\left\|y_{1}\right\|_{a} & \leq \max \left\{\bar{\gamma}_{1}\left(\left\|v_{1}\right\|_{a}\right), \bar{\gamma}_{1}^{u}\left(\left\|u_{1}\right\|_{a}\right)\right\} \\
\left\|y_{2[0, \infty)}\right\| & \leq \max \left\{\bar{\gamma}_{2}^{0}\left(\left\|x_{2}(0)\right\|\right), \bar{\gamma}_{2}\left(\left\|v_{2[0, \infty)}\right\|\right), \bar{\gamma}_{2}^{u}\left(\left\|u_{2[0, \infty)}\right\|\right)\right\} \\
\left\|y_{2}\right\|_{a} & \leq \max \left\{\bar{\gamma}_{2}\left(\left\|v_{2}\right\|_{a}\right), \bar{\gamma}_{2}^{u}\left(\left\|u_{2}\right\|_{a}\right)\right\}
\end{aligned}
$$

for some class $K$ functions $\bar{\gamma}_{1}^{0}, \bar{\gamma}_{2}^{0}, \bar{\gamma}_{1}, \bar{\gamma}_{2}, \bar{\gamma}_{1}^{u}$ and $\bar{\gamma}_{2}^{u}$.

Then if

$$
\bar{\gamma}_{1} \circ \bar{\gamma}_{2}(r)<r, \quad \forall r>0
$$

the system composed of (6) and (7) is ISS with restrictions $\tilde{X}_{1} \times \tilde{X}_{2}, \tilde{\Delta}_{1}$ and $\tilde{\Delta}_{2}$ on $x(0), u_{1}$ and $u_{2}$ respectively, viewing $x=\operatorname{col}\left(x_{1}, x_{2}\right)$ as state and $u=\operatorname{col}\left(u_{1}, u_{2}\right)$ as input, i.e., there exist class $K L$ function $\beta$ and class $K$ function $\gamma$, such that, for any initial state $x(0) \in \tilde{X}_{1} \times \tilde{X}_{2}$, and any input functions $u_{1}(t) \in L_{\infty}^{m_{1}}$ satisfying $\left\|u_{1[0, \infty)}\right\|<\tilde{\Delta}_{1}$ and $u_{2}(t) \in L_{\infty}^{m_{2}}$ satisfying $\left\|u_{2[0, \infty)}\right\|<\tilde{\Delta}_{2}$, the solution of (6) and (7) under connection (8) exists and satisfies, for all $t \geq 0$,

$$
\|x(t)\| \leq \max \left\{\beta(\|x(0)\|, t), \gamma\left(\left\|u_{[0, \infty)}\right\|\right)\right\}
$$

where,

$\tilde{X}_{1}=\left\{x_{1} \in X_{1}: \bar{\gamma}_{1}^{0}\left(\left\|x_{1}\right\|\right)<\Delta_{2}, \bar{\gamma}_{2} \circ \bar{\gamma}_{1}^{0}\left(\left\|x_{1}\right\|\right)<\Delta_{1}\right\}$

and 
$\tilde{X}_{2}=\left\{x_{2} \in X_{2}: \bar{\gamma}_{2}^{0}\left(\left\|x_{2}\right\|\right)<\Delta_{1}, \bar{\gamma}_{1} \circ \bar{\gamma}_{2}^{0}\left(\left\|x_{2}\right\|\right)<\Delta_{2}\right\}$

$\tilde{\Delta}_{1} \leq \Delta_{1}^{u}, \tilde{\Delta}_{2} \leq \Delta_{2}^{u}$

$s \in\left[0, \tilde{\Delta}_{1}\right) \Longrightarrow \bar{\gamma}_{2} \circ \bar{\gamma}_{1}^{u}(s)<\Delta_{1}, \bar{\gamma}_{1}^{u}(s)<\Delta_{2}$

and

$s \in\left[0, \tilde{\Delta}_{2}\right) \Longrightarrow \bar{\gamma}_{1} \circ \bar{\gamma}_{2}^{u}(s)<\Delta_{2}, \bar{\gamma}_{2}^{u}(s)<\Delta_{1}$.

REMARK 2.1. Theorem 2.1 is slightly different from Theorem B.3.1 [6] where for $i=1,2, \gamma_{i}(s)=\bar{\gamma}_{i}(s)$ and $\gamma_{i}^{u}(s)=\bar{\gamma}_{i}^{u}(s)$.

2.2. The Case of Uncertain Time-varying Nonlinear Systems. Let us first introduce a technical lemma which was established in [2].

Lemma 2.1. Let $\beta$ be a class $K L$ function, $\gamma$ a class $K$ function such that $\gamma(r)<r, \forall r>0$, and $\mu \in(0,1]$ a real number. For any nonnegative real numbers $s$ and $M$, and any nonnegative real function $z(t) \in L_{\infty}^{1}$ satisfying

$$
z(t) \leq \max \left\{\beta(s, t), \gamma\left(\left\|z_{[\mu t, t]}\right\|\right), M\right\}, \quad \forall t \geq 0,
$$

there exists a class $K_{\infty}$ function $\hat{\beta}$ such that

$$
z(t) \leq \max \{\hat{\beta}(s, t), M\}, \quad \forall t \geq 0 .
$$

Consider the interconnection of the following two systems as depicted in Figure 2 ,

$$
\begin{aligned}
& \dot{x}_{1}=f_{1}\left(x_{1}, v_{1}, u_{1}, d, t\right), \quad y_{1}=h_{1}\left(x_{1}, v_{1}, u_{1}, d, t\right) \\
& \dot{x}_{2}=f_{2}\left(x_{2}, v_{2}, u_{2}, d, t\right), \quad y_{2}=h_{2}\left(x_{2}, v_{2}, u_{2}, d, t\right)
\end{aligned}
$$

subject to the following interconnection:

$$
v_{1}=y_{2}, \quad v_{2}=y_{1}
$$

where, for $i=1,2, x_{i} \in \Re^{n_{i}}, u_{i} \in \Re^{m_{i}}, y_{i} \in \Re^{p_{i}}, v_{i} \in \Re^{q_{i}}$ with $p_{1}=q_{2}, p_{2}=q_{1}$, the functions $f_{1}\left(x_{1}, v_{1}, u_{1}, d, t\right)$ and $f_{2}\left(x_{2}, v_{2}, u_{2}, d, t\right)$ are piecewise continuous in $t$ and locally Lipschitz in $\operatorname{col}\left(x_{1}, v_{1}, u_{1}, d\right)$ and $\operatorname{col}\left(x_{2}, v_{2}, u_{2}, d\right)$ respectively, and $d$ : $\left[t_{0}, \infty\right) \mapsto \Re^{n_{d}}$ is piecewise continuous.

The system composed of (10) and (11) is interpreted as feedback interconnection of two subsystems, the upper one with state $x_{1}$, input $\operatorname{col}\left(v_{1}, u_{1}\right)$ and output $y_{1}$ and the lower one with state $x_{2}$, input $\operatorname{col}\left(v_{2}, u_{2}\right)$ and output $y_{2}$. And suppose the following assumption holds.

Assumption 2.2. The equations

$$
\begin{aligned}
& y_{1}=h_{1}\left(x_{1}, h_{2}\left(x_{2}, y_{1}, u_{2}, d, t\right), u_{1}, d, t\right) \\
& y_{2}=h_{2}\left(x_{2}, h_{1}\left(x_{1}, y_{2}, u_{1}, d, t\right), u_{2}, d, t\right)
\end{aligned}
$$




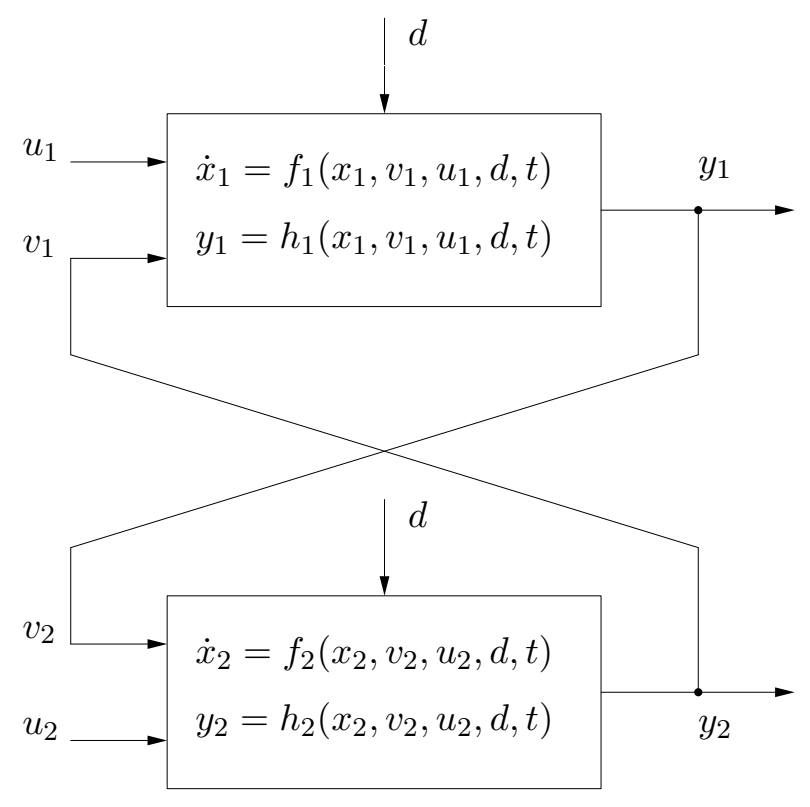

FIG. 2. Inter-connection of (10) and (11)

have a unique solution of the form $y=h(x, u, d, t)$ where $x=\operatorname{col}\left(x_{1}, x_{2}\right), y=$ $\operatorname{col}\left(y_{1}, y_{2}\right), u=\operatorname{col}\left(u_{1}, u_{2}\right)$, and $h$ is locally Lipschitz in $\operatorname{col}(x, u, d)$ and piecewise continuous in $t$.

Theorem 2.2. Assume that subsystem (10) is RISS with restrictions $X_{1}, \Delta_{1}$ and $\Delta_{1}^{u}$ on $x_{1}\left(t_{0}\right), v_{1}$ and $u_{1}$ respectively and subsystem (11) is RISS with restrictions $X_{2}, \Delta_{2}$ and $\Delta_{2}^{u}$ on $x_{2}\left(t_{0}\right), v_{2}$ and $u_{2}$ respectively, i.e., there exist class $K L$ functions $\beta_{1}$ and $\beta_{2}$, class $K$ functions $\gamma_{1}, \gamma_{1}^{u}, \gamma_{2}, \gamma_{2}^{u}$, independent of $d(t)$, such that, for any $x_{1}\left(t_{0}\right) \in X_{1}, v_{1}(t) \in L_{\infty}^{q_{1}}$ satisfying $\left\|v_{1\left[t_{0}, \infty\right)}\right\|<\Delta_{1}, u_{1}(t) \in L_{\infty}^{m_{1}}$ satisfying $\left\|u_{1\left[t_{0}, \infty\right)}\right\|<\Delta_{1}^{u}$, the solution of (10) exists and satisfies, for all $t \geq t_{0}$,

$$
\left\|x_{1}(t)\right\| \leq \max \left\{\beta_{1}\left(\left\|x_{1}\left(t_{0}\right)\right\|, t-t_{0}\right), \gamma_{1}\left(\left\|v_{1\left[t_{0}, t\right]}\right\|\right), \gamma_{1}^{u}\left(\left\|u_{1\left[t_{0}, t\right]}\right\|\right)\right\}
$$

and for any $x_{2}\left(t_{0}\right) \in X_{2}, v_{2}(t) \in L_{\infty}^{q_{2}}$ satisfying $\left\|v_{2\left[t_{0}, \infty\right)}\right\|<\Delta_{2}, u_{2}(t) \in L_{\infty}^{m_{2}}$ satisfying $\left\|u_{2\left[t_{0}, \infty\right)}\right\|<\Delta_{2}^{u}$, the solution of (11) exists and satisfies, for all $t \geq t_{0}$,

$$
\left\|x_{2}(t)\right\| \leq \max \left\{\beta_{2}\left(\left\|x_{2}\left(t_{0}\right)\right\|, t-t_{0}\right), \gamma_{2}\left(\left\|v_{2\left[t_{0}, t\right]}\right\|\right), \gamma_{2}^{u}\left(\left\|u_{2\left[t_{0}, t\right]}\right\|\right)\right\} .
$$

Further assume that subsystem (10) is RIOS with restrictions $\bar{X}_{1}, \bar{\Delta}_{1}$ and $\bar{\Delta}_{1}^{u}$ on $x_{1}\left(t_{0}\right), v_{1}$ and $u_{1}$ respectively and subsystem (11) is RIOS with restrictions $\bar{X}_{2}, \bar{\Delta}_{2}$ and $\bar{\Delta}_{2}^{u}$ on $x_{2}\left(t_{0}\right), v_{2}$ and $u_{2}$ respectively, i.e., there exist class $K L$ functions $\bar{\beta}_{1}$ and $\bar{\beta}_{2}$, 
class $K$ functions $\bar{\gamma}_{1}, \bar{\gamma}_{1}^{u}, \bar{\gamma}_{2}, \bar{\gamma}_{2}^{u}$, independent of $d(t)$, such that, for any $x_{1}\left(t_{0}\right) \in \bar{X}_{1}$, $v_{1}(t) \in L_{\infty}^{q_{1}}$ satisfying $\left\|v_{1\left[t_{0}, \infty\right)}\right\|<\bar{\Delta}_{1}, u_{1}(t) \in L_{\infty}^{m_{1}}$ satisfying $\left\|u_{1\left[t_{0}, \infty\right)}\right\|<\bar{\Delta}_{1}^{u}$, the output of (10) exists and satisfies, for all $t \geq t_{0}$,

$$
\left\|y_{1}(t)\right\| \leq \max \left\{\bar{\beta}_{1}\left(\left\|x_{1}\left(t_{0}\right)\right\|, t-t_{0}\right), \bar{\gamma}_{1}\left(\left\|v_{1\left[t_{0}, t\right]}\right\|\right), \bar{\gamma}_{1}^{u}\left(\left\|u_{1\left[t_{0}, t\right]}\right\|\right)\right\}
$$

and for any $x_{2}\left(t_{0}\right) \in \bar{X}_{2}, v_{2}(t) \in L_{\infty}^{q_{2}}$ satisfying $\left\|v_{2\left[t_{0}, \infty\right)}\right\|<\bar{\Delta}_{2}, u_{2}(t) \in L_{\infty}^{m_{2}}$ satisfying $\left\|u_{2\left[t_{0}, \infty\right)}\right\|<\bar{\Delta}_{2}^{u}$, the output of (11) exists and satisfies, for all $t \geq t_{0}$,

$$
\left\|y_{2}(t)\right\| \leq \max \left\{\bar{\beta}_{2}\left(\left\|x_{2}\left(t_{0}\right)\right\|, t-t_{0}\right), \bar{\gamma}_{2}\left(\left\|v_{2\left[t_{0}, t\right]}\right\|\right), \bar{\gamma}_{2}^{u}\left(\left\|u_{2\left[t_{0}, t\right]}\right\|\right)\right\} .
$$

Suppose that the small gain condition

$$
\bar{\gamma}_{1} \circ \bar{\gamma}_{2}(r)<r, \quad r>0
$$

holds. Then the system composed of (10) and (11) with connection (12) is RISS and RIOS with restrictions $\tilde{X}_{1} \times \tilde{X}_{2}, \tilde{\Delta}_{1}$ and $\tilde{\Delta}_{2}$ on $x\left(t_{0}\right), u_{1}$ and $u_{2}$ respectively, viewing $x=\operatorname{col}\left(x_{1}, x_{2}\right)$ as state, $y=\operatorname{col}\left(y_{1}, y_{2}\right)$ as output and $u=\operatorname{col}\left(u_{1}, u_{2}\right)$ as input, i.e., there exist class $K L$ functions $\beta$ and $\bar{\beta}$, class $K$ functions $\gamma$ and $\bar{\gamma}$, independent of $d(t)$, such that, for any initial state $x\left(t_{0}\right) \in \tilde{X}_{1} \times \tilde{X}_{2}$, and any input functions $u_{1}(t) \in L_{\infty}^{m_{1}}$ satisfying $\left\|u_{1\left[t_{0}, \infty\right)}\right\|<\tilde{\Delta}_{1}$ and $u_{2}(t) \in L_{\infty}^{m_{2}}$ satisfying $\left\|u_{2\left[t_{0}, \infty\right)}\right\|<\tilde{\Delta}_{2}$, the solution and output of (10) and (11) with connection (12) exist and satisfy, for all $t \geq t_{0}$,

$$
\begin{aligned}
\|x(t)\| & \leq \max \left\{\beta\left(\left\|x\left(t_{0}\right)\right\|, t-t_{0}\right), \gamma\left(\left\|u_{\left[t_{0}, t\right]}\right\|\right)\right\} \\
\|y(t)\| & \leq \max \left\{\bar{\beta}\left(\left\|x\left(t_{0}\right)\right\|, t-t_{0}\right), \bar{\gamma}\left(\left\|u_{\left[t_{0}, t\right]}\right\|\right)\right\}
\end{aligned}
$$

where,

$\gamma(s)=\max \left\{4 \gamma_{1} \circ \bar{\gamma}_{1} \circ \bar{\gamma}_{2}^{u}(s), 4 \gamma_{1} \circ \bar{\gamma}_{1}^{u}(s), 4 \gamma_{1} \circ \bar{\gamma}_{2} \circ \bar{\gamma}_{1}^{u}(s), 4 \gamma_{1} \circ \bar{\gamma}_{2}^{u}(s), 2 \gamma_{1}^{u}(s), 4 \gamma_{2} \circ \bar{\gamma}_{1} \circ\right.$ $\left.\bar{\gamma}_{2}^{u}(s), 4 \gamma_{2} \circ \bar{\gamma}_{1}^{u}(s), 4 \gamma_{2} \circ \bar{\gamma}_{2} \circ \bar{\gamma}_{1}^{u}(s), 4 \gamma_{2} \circ \bar{\gamma}_{2}^{u}(s), 2 \gamma_{2}^{u}(s)\right\}$,

$\bar{\gamma}(s)=\max \left\{2 \bar{\gamma}_{1} \circ \bar{\gamma}_{2}^{u}(s), 2 \bar{\gamma}_{1}^{u}(s), 2 \bar{\gamma}_{2} \circ \bar{\gamma}_{1}^{u}(s), 2 \bar{\gamma}_{2}^{u}(s)\right\}$

and,

(i) If $\Delta_{1}, \Delta_{2}, \bar{\Delta}_{1}, \bar{\Delta}_{2}$ are finite,

$\tilde{X}_{1}=\left\{x_{1} \in X_{1} \cap \bar{X}_{1}: \bar{\beta}_{1}\left(\left\|x_{1}\right\|, 0\right)<\min \left\{\Delta_{2}, \bar{\Delta}_{2}\right\}, \bar{\gamma}_{2} \circ \bar{\beta}_{1}\left(\left\|x_{1}\right\|, 0\right)<\min \left\{\Delta_{1}, \bar{\Delta}_{1}\right\}\right\}$

and

$\tilde{X}_{2}=\left\{x_{2} \in X_{2} \cap \bar{X}_{2}: \bar{\beta}_{2}\left(\left\|x_{2}\right\|, 0\right)<\min \left\{\Delta_{1}, \bar{\Delta}_{1}\right\}, \bar{\gamma}_{1} \circ \bar{\beta}_{2}\left(\left\|x_{2}\right\|, 0\right)<\min \left\{\Delta_{2}, \bar{\Delta}_{2}\right\}\right\}$.

$\tilde{\Delta}_{1} \leq \min \left\{\Delta_{1}^{u}, \bar{\Delta}_{1}^{u}\right\}, \tilde{\Delta}_{2} \leq \min \left\{\Delta_{2}^{u}, \bar{\Delta}_{2}^{u}\right\}$

$s \in\left[0, \tilde{\Delta}_{1}\right) \Longrightarrow \bar{\gamma}_{2} \circ \bar{\gamma}_{1}^{u}(s)<\min \left\{\Delta_{1}, \bar{\Delta}_{1}\right\}, \bar{\gamma}_{1}^{u}(s)<\min \left\{\Delta_{2}, \bar{\Delta}_{2}\right\}$

and

$s \in\left[0, \tilde{\Delta}_{2}\right) \Longrightarrow \bar{\gamma}_{1} \circ \bar{\gamma}_{2}^{u}(s)<\min \left\{\Delta_{2}, \bar{\Delta}_{2}\right\}, \bar{\gamma}_{2}^{u}(s)<\min \left\{\Delta_{1}, \bar{\Delta}_{1}\right\}$.

(ii) If $\Delta_{1}, \Delta_{2}, \bar{\Delta}_{1}, \bar{\Delta}_{2}$ are infinite,

$\tilde{X}_{1}=X_{1} \cap \bar{X}_{1}, \tilde{X}_{2}=X_{2} \cap \bar{X}_{2}$ 
and

$\tilde{\Delta}_{1} \leq \min \left\{\Delta_{1}^{u}, \bar{\Delta}_{1}^{u}\right\}, \tilde{\Delta}_{2} \leq \min \left\{\Delta_{2}^{u}, \bar{\Delta}_{2}^{u}\right\}$.

Proof. First it is noted that the inequality $\bar{\gamma}_{1} \circ \bar{\gamma}_{2}(r)<r, r>0$ and the following one,

$$
\bar{\gamma}_{2} \circ \bar{\gamma}_{1}(r)<r, \quad r>0
$$

imply each other [5].

Step1: In this step, we will show that if $x_{1}\left(t_{0}\right) \in X_{1} \cap \bar{X}_{1}, x_{2}\left(t_{0}\right) \in X_{2} \cap \bar{X}_{2}, u_{1}(t) \in$ $L_{\infty}^{m_{1}}$ satisfying $\left\|u_{1\left[t_{0}, \infty\right)}\right\|<\min \left\{\Delta_{1}^{u}, \bar{\Delta}_{1}^{u}\right\}$, and $u_{2}(t) \in L_{\infty}^{m_{2}}$ satisfying $\left\|u_{2\left[t_{0}, \infty\right)}\right\|<$ $\min \left\{\Delta_{2}^{u}, \bar{\Delta}_{2}^{u}\right\}$, the solution of the inter-connected system exists and is bounded for all $t \geq t_{0}$. For this purpose, we will consider the following two cases.

(i) $\Delta_{1}, \Delta_{2}, \bar{\Delta}_{1}$ and $\bar{\Delta}_{2}$ are infinite.

Toward this end, we will first prove that the outputs $y_{1}$ and $y_{2}$ exist for all $t \geq t_{0}$ and are bounded in a standard way such as the proof of Theorem 10.6.1 [5]. Suppose this is not the case, for every number $R>0$, there exists a time $T>t_{0}$ such that the solutions are defined on $[0, T]$ and either $\left\|y_{1}(T)\right\| \geq R$ or $\left\|y_{2}(T)\right\| \geq R$.

Without loss of generality, we only consider the case where $\left\|y_{1}(T)\right\| \geq R$. Choose $R$ such that

$R>\max \left\{\bar{\beta}_{1}\left(r_{1}, 0\right), \bar{\gamma}_{1} \circ \bar{\beta}_{2}\left(r_{2}, 0\right), \bar{\gamma}_{1}^{u}\left(\Delta_{1}^{u}\right), \bar{\gamma}_{1}^{u}\left(\bar{\Delta}_{1}^{u}\right), \bar{\gamma}_{1} \circ \bar{\gamma}_{2}^{u}\left(\Delta_{2}^{u}\right), \bar{\gamma}_{1} \circ \bar{\gamma}_{2}^{u}\left(\bar{\Delta}_{2}^{u}\right)\right\}$, where, $r_{1}=\left\{x_{1} \in X_{1} \cap \bar{X}_{1}: \sup \left(\left\|x_{1}\right\|\right)\right\}, r_{2}=\left\{x_{2} \in X_{2} \cap \bar{X}_{2}: \sup \left(\left\|x_{2}\right\|\right)\right\}$.

It follows from (15) and (16) that

$$
\begin{aligned}
\left\|y_{1\left[t_{0}, T\right]}\right\| & \leq \max \left\{\bar{\beta}_{1}\left(\left\|x_{1}\left(t_{0}\right)\right\|, 0\right), \bar{\gamma}_{1}\left(\left\|y_{2\left[t_{0}, T\right]}\right\|\right), \bar{\gamma}_{1}^{u}\left(\left\|u_{1\left[t_{0}, T\right]}\right\|\right)\right\} \\
\left\|y_{2\left[t_{0}, T\right]}\right\| & \leq \max \left\{\bar{\beta}_{2}\left(\left\|x_{2}\left(t_{0}\right)\right\|, 0\right), \bar{\gamma}_{2}\left(\left\|y_{1\left[t_{0}, T\right]}\right\|\right), \bar{\gamma}_{2}^{u}\left(\left\|u_{2\left[t_{0}, T\right]}\right\|\right)\right\} .
\end{aligned}
$$

Substituting (19) into (18) gives that

$$
\begin{aligned}
\left\|y_{1\left[t_{0}, T\right]}\right\| \leq & \max \left\{\bar{\beta}_{1}\left(\left\|x_{1}\left(t_{0}\right)\right\|, 0\right), \bar{\gamma}_{1} \circ \bar{\beta}_{2}\left(\left\|x_{2}\left(t_{0}\right)\right\|, 0\right),\right. \\
& \left.\bar{\gamma}_{1} \circ \bar{\gamma}_{2}\left(\left\|y_{1\left[t_{0}, T\right]}\right\|\right), \bar{\gamma}_{1} \circ \bar{\gamma}_{2}^{u}\left(\left\|u_{2\left[t_{0}, T\right]}\right\|\right), \bar{\gamma}_{1}^{u}\left(\left\|u_{1\left[t_{0}, T\right]}\right\|\right)\right\}
\end{aligned}
$$

Since

$$
\bar{\gamma}_{1} \circ \bar{\gamma}_{2}\left(\left\|y_{1\left[t_{0}, T\right]}\right\|\right)<\left\|y_{1\left[t_{0}, T\right]}\right\|
$$

it holds that

$$
\begin{aligned}
\left\|y_{1\left[t_{0}, T\right]}\right\| \leq & \max \left\{\bar{\beta}_{1}\left(\left\|x_{1}\left(t_{0}\right)\right\|, 0\right), \bar{\gamma}_{1} \circ \bar{\beta}_{2}\left(\left\|x_{2}\left(t_{0}\right)\right\|, 0\right),\right. \\
& \left.\bar{\gamma}_{1} \circ \bar{\gamma}_{2}^{u}\left(\left\|u_{2\left[t_{0}, T\right]}\right\|\right), \bar{\gamma}_{1}^{u}\left(\left\|u_{1\left[t_{0}, T\right]}\right\|\right)\right\}<R
\end{aligned}
$$


which contradicts $\left\|y_{1}(T)\right\|>R$. Therefore the outputs are bounded for all $t \geq t_{0}$.

Since the subsystems (10) and (11) are RISS with restrictions, the solution of the inter-connected system is bounded for all $t \geq t_{0}$.

(ii) At least one of $\Delta_{1}, \Delta_{2}, \bar{\Delta}_{1}$ and $\bar{\Delta}_{2}$ is finite.

Toward this end, we will first prove that the outputs $y_{1}$ and $y_{2}$ exist for all $t \geq t_{0}$ and are bounded in a way motivated from the proof of Theorem 1 [20].

For any given $x\left(t_{0}\right) \in \tilde{X}_{1} \times \tilde{X}_{2}$, let $p\left(x\left(t_{0}\right), \lambda\right)$ be a continuous path in $\tilde{X}_{1} \times \tilde{X}_{2}$ from the origin to $x\left(t_{0}\right)$ with the property that $p\left(x\left(t_{0}\right), 0\right)$ is the origin and $p\left(x\left(t_{0}\right), 1\right)=$ $x\left(t_{0}\right)$, and let $y_{1}^{\lambda}$ and $y_{2}^{\lambda}$ be the outputs starting at $x^{\lambda}\left(t_{0}\right)=p\left(x\left(t_{0}\right), \lambda\right)$ with inputs $\lambda u_{1}$ and $\lambda u_{2}$. When $\lambda=0$, the solutions and outputs are defined on $\left[t_{0}, \infty\right)$ and identically zero. Note that the solutions are continuous functions of $\lambda$. Hence, for any given $T>t_{0}$ (arbitrarily large), $\epsilon_{1}>0$ and $\epsilon_{2}>0$, there exists $\lambda^{*}$ such that the solution exists on $\left[t_{0}, T\right]$ and

$$
\left\|y_{1\left[t_{0}, T\right]}^{\lambda}\right\| \leq \epsilon_{1}, \quad\left\|y_{2\left[t_{0}, T\right]}^{\lambda}\right\| \leq \epsilon_{2}
$$

for all $\lambda \in\left[0, \lambda^{*}\right]$.

Denote that

$$
\begin{aligned}
\bar{\Delta}_{1}=\max & \left\{\bar{\beta}_{1}\left(\max _{\lambda \in[0,1]}\left\|x_{1}^{\lambda}\left(t_{0}\right)\right\|, 0\right), \bar{\gamma}_{1} \circ \bar{\beta}_{2}\left(\max _{\lambda \in[0,1]}\left\|x_{2}^{\lambda}\left(t_{0}\right)\right\|, 0\right),\right. \\
& \left.\bar{\gamma}_{1} \circ \bar{\gamma}_{2}^{u}\left(\left\|u_{2\left[t_{0}, \infty\right)}\right\|\right), \bar{\gamma}_{1}^{u}\left(\left\|u_{1\left[t_{0}, \infty\right)}\right\|\right)\right\}, \\
\bar{\Delta}_{2}=\max \{ & \left\{\bar{\beta}_{2}\left(\max _{\lambda \in[0,1]}\left\|x_{2}^{\lambda}\left(t_{0}\right)\right\|, 0\right), \bar{\gamma}_{2} \circ \bar{\beta}_{1}\left(\max _{\lambda \in[0,1]}\left\|x_{1}^{\lambda}\left(t_{0}\right)\right\|, 0\right),\right. \\
& \left.\bar{\gamma}_{2} \circ \bar{\gamma}_{1}^{u}\left(\left\|u_{1\left[t_{0}, \infty\right)}\right\|\right), \bar{\gamma}_{2}^{u}\left(\left\|u_{2\left[t_{0}, \infty\right)}\right\|\right)\right\} .
\end{aligned}
$$

Since $p\left(x\left(t_{0}\right), \lambda\right)$ belongs to $\tilde{X}_{1} \times \tilde{X}_{2}$ and $\left\|u_{1\left[t_{0}, \infty\right)}\right\|<\tilde{\Delta}_{1},\left\|u_{2\left[t_{0}, \infty\right)}\right\|<\tilde{\Delta}_{2}$, it holds that $\bar{\Delta}_{1}<\min \left\{\Delta_{2}, \bar{\Delta}_{2}\right\}$ and $\bar{\Delta}_{2}<\min \left\{\Delta_{1}, \bar{\Delta}_{1}\right\}$. Let $T>t_{0}$ be arbitrarily large and $\epsilon_{1}, \epsilon_{2}$ satisfy $\bar{\Delta}_{1}<\epsilon_{1}<\min \left\{\Delta_{2}, \bar{\Delta}_{2}\right\}, \bar{\Delta}_{2}<\epsilon_{2}<\min \left\{\Delta_{1}, \bar{\Delta}_{1}\right\}$, and let $\lambda^{*} \in(0,1]$ be the largest value such that (22) holds for all $\lambda \in\left[0, \lambda^{*}\right]$. Suppose $\lambda^{*}<1$. Since $\left\|y_{1\left[t_{0}, T\right]}^{\lambda}\right\|<\min \left\{\Delta_{2}, \bar{\Delta}_{2}\right\}$ and $\left\|y_{2\left[t_{0}, T\right]}^{\lambda}\right\|<\min \left\{\Delta_{1}, \bar{\Delta}_{1}\right\}$, following the same lines as $(i)$ when $\Delta_{1}, \Delta_{2}, \bar{\Delta}_{1}$ and $\bar{\Delta}_{2}$ are infinite, we have that

$$
\left\|y_{1\left[t_{0}, T\right]}^{\lambda}\right\| \leq \bar{\Delta}_{1}<\epsilon_{1}, \quad\left\|y_{2\left[t_{0}, T\right]}^{\lambda}\right\| \leq \bar{\Delta}_{2}<\epsilon_{2} .
$$

By continuity of solutions, there exists $\lambda^{\prime}>\lambda^{*}$ such that (22) holds, contradicting that $\lambda^{*}<1$. Hence $\lambda^{*}=1$. Since $T$ can be arbitrarily large, $\left\|y_{1\left[t_{0}, \infty\right)}\right\|<\min \left\{\Delta_{2}, \bar{\Delta}_{2}\right\}$ and $\left\|y_{2\left[t_{0}, \infty\right)}\right\|<\min \left\{\Delta_{1}, \bar{\Delta}_{1}\right\}$.

In both cases, the solution of the inter-connected system exist and is bounded for all $t \geq t_{0}$. Moreover, $\left\|y_{1\left[t_{0}, \infty\right)}\right\|<\min \left\{\Delta_{2}, \bar{\Delta}_{2}\right\}$ and $\left\|y_{2\left[t_{0}, \infty\right)}\right\|<\min \left\{\Delta_{1}, \bar{\Delta}_{1}\right\}$. 
Step2: We will show the system composed of (10) and (11) is RIOS with restrictions $\tilde{X}_{1} \times \tilde{X}_{2}, \tilde{\Delta}_{1}$ and $\tilde{\Delta}_{2}$ on $x\left(t_{0}\right), u_{1}$ and $u_{2}$ respectively, viewing $x=\operatorname{col}\left(x_{1}, x_{2}\right)$ as state, $y=\operatorname{col}\left(y_{1}, y_{2}\right)$ as output and $u=\operatorname{col}\left(u_{1}, u_{2}\right)$ as input.

By symmetry of $y_{1}$ and $y_{2}$, it follows from (21) that

$$
\begin{aligned}
& \left\|y_{1\left[t_{0}, \infty\right)}\right\| \leq \max \left\{\bar{\beta}_{1}\left(\left\|x_{1}\left(t_{0}\right)\right\|, 0\right), \bar{\gamma}_{1} \circ \bar{\beta}_{2}\left(\left\|x_{2}\left(t_{0}\right)\right\|, 0\right),\right. \\
& \left.\bar{\gamma}_{1} \circ \bar{\gamma}_{2}^{u}\left(\left\|u_{2\left[t_{0}, \infty\right)}\right\|\right), \bar{\gamma}_{1}^{u}\left(\left\|u_{1\left[t_{0}, \infty\right)}\right\|\right)\right\} \\
& \leq \max \left\{\delta_{1}\left(\left\|x\left(t_{0}\right)\right\|\right), M_{1}\right\} \\
& \left\|y_{2\left[t_{0}, \infty\right)}\right\| \leq \max \left\{\bar{\beta}_{2}\left(\left\|x_{2}\left(t_{0}\right)\right\|, 0\right), \bar{\gamma}_{2} \circ \bar{\beta}_{1}\left(\left\|x_{1}\left(t_{0}\right)\right\|, 0\right),\right. \\
& \left.\bar{\gamma}_{2} \circ \bar{\gamma}_{1}^{u}\left(\left\|u_{1\left[t_{0}, \infty\right)}\right\|\right), \bar{\gamma}_{2}^{u}\left(\left\|u_{2\left[t_{0}, \infty\right)}\right\|\right)\right\} \\
& \leq \max \left\{\delta_{2}\left(\left\|x\left(t_{0}\right)\right\|\right), M_{2}\right\}
\end{aligned}
$$

where,

$$
\begin{aligned}
& \delta_{1}(s)=\max \left\{\bar{\beta}_{1}(s, 0), \bar{\gamma}_{1} \circ \bar{\beta}_{2}(s, 0)\right\}, \quad \delta_{2}(s)=\max \left\{\bar{\beta}_{2}(s, 0), \bar{\gamma}_{2} \circ \bar{\beta}_{1}(s, 0)\right\}, \\
& M_{1}=\max \left\{\bar{\gamma}_{1} \circ \bar{\gamma}_{2}^{u}\left(\left\|u_{2\left[t_{0}, \infty\right)}\right\|\right), \bar{\gamma}_{1}^{u}\left(\left\|u_{1\left[t_{0}, \infty\right)}\right\|\right)\right\}, \\
& M_{2}=\max \left\{\bar{\gamma}_{2} \circ \bar{\gamma}_{1}^{u}\left(\left\|u_{1\left[t_{0}, \infty\right)}\right\|\right), \bar{\gamma}_{2}^{u}\left(\left\|u_{2\left[t_{0}, \infty\right)}\right\|\right)\right\} .
\end{aligned}
$$

Hence,

$$
\begin{aligned}
\|y(t)\| & \leq\left\|y_{1\left[t_{0}, \infty\right)}\right\|+\left\|y_{2\left[t_{0}, \infty\right)}\right\| \\
& \leq \max \left\{2 \delta_{1}\left(\left\|x\left(t_{0}\right)\right\|\right), 2 \delta_{2}\left(\left\|x\left(t_{0}\right)\right\|\right), 2 M_{1}, 2 M_{2}\right\} \\
& \leq \max \left\{\delta_{3}\left(\left\|x\left(t_{0}\right)\right\|\right), M_{3}\right\} \stackrel{\text { def }}{=} y_{\infty}
\end{aligned}
$$

where, $\delta_{3}=\max \left\{2 \delta_{1}(s), 2 \delta_{2}(s)\right\}$ and $M_{3}=\bar{\gamma}\left(\left\|u_{\left[t_{0}, \infty\right)}\right\|\right)$ for any $K_{\infty}$ function $\bar{\gamma}$ satisfying

$$
\bar{\gamma}(s) \geq \max \left\{2 \bar{\gamma}_{1} \circ \bar{\gamma}_{2}^{u}(s), 2 \bar{\gamma}_{1}^{u}(s), 2 \bar{\gamma}_{2} \circ \bar{\gamma}_{1}^{u}(s), 2 \bar{\gamma}_{2}^{u}(s)\right\} .
$$

Relying upon (23) and (24), the restrictions $\tilde{X}_{1} \times \tilde{X}_{2}$ on the initial state $x\left(t_{0}\right)$ and $\tilde{\Delta}_{1}, \tilde{\Delta}_{2}$ on the inputs $u_{1}, u_{2}$ respectively can be computed as follows:

(i) If $\Delta_{1}, \Delta_{2}, \bar{\Delta}_{1}, \bar{\Delta}_{2}$ are finite,

$\tilde{X}_{1}=\left\{x_{1} \in X_{1} \cap \bar{X}_{1}: \bar{\beta}_{1}\left(\left\|x_{1}\right\|, 0\right)<\min \left\{\Delta_{2}, \bar{\Delta}_{2}\right\}, \bar{\gamma}_{2} \circ \bar{\beta}_{1}\left(\left\|x_{1}\right\|, 0\right)<\min \left\{\Delta_{1}, \bar{\Delta}_{1}\right\}\right\}$ and

$\tilde{X}_{2}=\left\{x_{2} \in X_{2} \cap \bar{X}_{2}: \bar{\beta}_{2}\left(\left\|x_{2}\right\|, 0\right)<\min \left\{\Delta_{1}, \bar{\Delta}_{1}\right\}, \bar{\gamma}_{1} \circ \bar{\beta}_{2}\left(\left\|x_{2}\right\|, 0\right)<\min \left\{\Delta_{2}, \bar{\Delta}_{2}\right\}\right\}$.

$\tilde{\Delta}_{1} \leq \min \left\{\Delta_{1}^{u}, \bar{\Delta}_{1}^{u}\right\}, \tilde{\Delta}_{2} \leq \min \left\{\Delta_{2}^{u}, \bar{\Delta}_{2}^{u}\right\}$

$s \in\left[0, \tilde{\Delta}_{1}\right) \Longrightarrow \bar{\gamma}_{2} \circ \bar{\gamma}_{1}^{u}(s)<\min \left\{\Delta_{1}, \bar{\Delta}_{1}\right\}, \bar{\gamma}_{1}^{u}(s)<\min \left\{\Delta_{2}, \bar{\Delta}_{2}\right\}$

and

$s \in\left[0, \tilde{\Delta}_{2}\right) \Longrightarrow \bar{\gamma}_{1} \circ \bar{\gamma}_{2}^{u}(s)<\min \left\{\Delta_{2}, \bar{\Delta}_{2}\right\}, \bar{\gamma}_{2}^{u}(s)<\min \left\{\Delta_{1}, \bar{\Delta}_{1}\right\}$ 
(ii) If $\Delta_{1}, \Delta_{2}, \bar{\Delta}_{1}, \bar{\Delta}_{2}$ are infinite,

$\tilde{X}_{1}=X_{1} \cap \bar{X}_{1}, \tilde{X}_{2}=X_{2} \cap \bar{X}_{2}$

and

$\tilde{\Delta}_{1} \leq \min \left\{\Delta_{1}^{u}, \bar{\Delta}_{1}^{u}\right\}, \tilde{\Delta}_{2} \leq \min \left\{\Delta_{2}^{u}, \bar{\Delta}_{2}^{u}\right\}$.

From (13) and (14), we could obtain that

$$
\begin{aligned}
\|x(t)\| & \leq\left\|x_{1}(t)\right\|+\left\|x_{2}(t)\right\| \\
& \leq \max \left\{\alpha_{x}\left(\left\|x\left(t_{0}\right)\right\|\right), \alpha_{u}\left(\left\|u_{\left[t_{0}, t\right]}\right\|\right), \alpha_{y}\left(\left\|y_{\left[t_{0}, t\right]}\right\|\right)\right\} \\
& \leq \max \left\{\alpha_{x}\left(\left\|x\left(t_{0}\right)\right\|\right), \alpha_{u}\left(\left\|u_{\left[t_{0}, \infty\right)}\right\|\right), \alpha_{y}\left(y_{\infty}\right)\right\} \stackrel{\text { def }}{=} x_{\infty}
\end{aligned}
$$

where $\alpha_{x}(s)=\max \left\{2 \beta_{1}(s, 0), 2 \beta_{2}(s, 0)\right\}, \alpha_{u}(s)=\max \left\{2 \gamma_{1}^{u}(s), 2 \gamma_{2}^{u}(s)\right\}, \alpha_{y}(s)=$ $\max \left\{2 \gamma_{1}(s), 2 \gamma_{2}(s)\right\}$.

For any time $t_{1} \geq 0$, it holds that

$$
\begin{aligned}
\left\|y_{1}\left(t_{0}+t_{1}\right)\right\| \leq \max & \left\{\bar{\beta}_{1}\left(\left\|x_{1}\left(t_{0}+\frac{t_{1}}{2}\right)\right\|, \frac{t_{1}}{2}\right), \bar{\gamma}_{1}\left(\left\|y_{2\left[t_{0}+\frac{t_{1}}{2}, t_{0}+t_{1}\right]}\right\|\right),\right. \\
& \left.\bar{\gamma}_{1}^{u}\left(\left\|u_{1\left[t_{0}+\frac{t_{1}}{2}, t_{0}+t_{1}\right]}\right\|\right)\right\} \\
\leq & \max \left\{\bar{\beta}_{1}\left(x_{\infty}, \frac{t_{1}}{2}\right), \bar{\gamma}_{1}\left(\left\|y_{2\left[t_{0}+\frac{t_{1}}{2}, t_{0}+t_{1}\right]}\right\|\right), \bar{\gamma}_{1}^{u}\left(\left\|u_{1\left[t_{0}, \infty\right)}\right\|\right)\right\}
\end{aligned}
$$

and for $\tau \in\left[\frac{t_{1}}{2}, t_{1}\right]$, it follows that

$$
\begin{aligned}
\left\|y_{2}\left(t_{0}+\tau\right)\right\| \leq & \max \left\{\bar{\beta}_{2}\left(\left\|x_{2}\left(t_{0}+\frac{t_{1}}{4}\right)\right\|, \tau-\frac{t_{1}}{4}\right), \bar{\gamma}_{2}\left(\left\|y_{1\left[t_{0}+\frac{t_{1}}{4}, t_{0}+\tau\right]}\right\|\right),\right. \\
& \left.\bar{\gamma}_{2}^{u}\left(\left\|u_{2\left[t_{0}+\frac{t_{1}}{4}, t_{0}+\tau\right]}\right\|\right)\right\} \\
& \leq \max \left\{\bar{\beta}_{2}\left(x_{\infty}, \frac{t_{1}}{4}\right), \bar{\gamma}_{2}\left(\left\|y_{1\left[t_{0}+\frac{t_{1}}{4}, t_{0}+t_{1}\right]}\right\|\right), \bar{\gamma}_{2}^{u}\left(\left\|u_{2\left[t_{0}, \infty\right)}\right\|\right)\right\} .
\end{aligned}
$$

Substituting (27) into (26) gives that

$$
\begin{aligned}
\left\|y_{1}\left(t_{0}+t_{1}\right)\right\| \leq & \max \left\{\bar{\beta}_{1}\left(x_{\infty}, \frac{t_{1}}{2}\right), \bar{\gamma}_{1} \circ \bar{\beta}_{2}\left(x_{\infty}, \frac{t_{1}}{4}\right), \bar{\gamma}_{1} \circ \bar{\gamma}_{2}\left(\left\|y_{1\left[t_{0}+\frac{t_{1}}{4}, t_{0}+t_{1}\right]}\right\|\right),\right. \\
& \left.\bar{\gamma}_{1} \circ \bar{\gamma}_{2}^{u}\left(\left\|u_{2\left[t_{0}, \infty\right)}\right\|\right), \bar{\gamma}_{1}^{u}\left(\left\|u_{1\left[t_{0}, \infty\right)}\right\|\right)\right\} \\
\leq & \max \left\{\tilde{\beta}_{1}\left(x_{\infty}, t_{1}\right), \bar{\gamma}_{1} \circ \bar{\gamma}_{2}\left(\left\|y_{1\left[t_{0}+\frac{t_{1}}{4}, t_{0}+t_{1}\right]}\right\|\right), M_{1}\right\}
\end{aligned}
$$

for any class $K L$ function $\tilde{\beta}_{1}$ satisfying

$$
\tilde{\beta}_{1}(s, t) \geq \max \left\{\bar{\beta}_{1}\left(s, \frac{t}{2}\right), \bar{\gamma}_{1} \circ \bar{\beta}_{2}\left(s, \frac{t}{4}\right)\right\} .
$$

Denoting $z_{1}\left(t_{1}\right)=\left\|y_{1}\left(t_{0}+t_{1}\right)\right\|$ gives that

$$
z_{1}\left(t_{1}\right) \leq \max \left\{\tilde{\beta}_{1}\left(x_{\infty}, t_{1}\right), \bar{\gamma}_{1} \circ \bar{\gamma}_{2}\left(\left\|z_{1\left[\frac{t_{1}}{4}, t_{1}\right]}\right\|\right), M_{1}\right\} .
$$

Since $\bar{\gamma}_{1} \circ \bar{\gamma}_{2}(r)<r(r>0)$, we invoke Lemma 2.1 to conclude that there exists a class $K L$ function $\hat{\beta}_{1}$ such that $z_{1}\left(t_{1}\right) \leq \max \left\{\hat{\beta}_{1}\left(x_{\infty}, t_{1}\right), M_{1}\right\}$. It follows that

$$
\left\|y_{1}(t)\right\| \leq \max \left\{\hat{\beta}_{1}\left(x_{\infty}, t-t_{0}\right), M_{1}\right\} .
$$


By symmetry of $y_{1}$ and $y_{2}$, there exists some class $K L$ function $\hat{\beta}_{2}$ such that

$$
\left\|y_{2}(t)\right\| \leq \max \left\{\hat{\beta}_{2}\left(x_{\infty}, t-t_{0}\right), M_{2}\right\} .
$$

Toward this end, consider the following two cases of $y_{\infty}$ in (25).

(i) $\delta_{3}\left(\left\|x\left(t_{0}\right)\right\|\right) \geq M_{3}$ : We have $y_{\infty}=\delta_{3}\left(\left\|x\left(t_{0}\right)\right\|\right)$, and $\left\|u_{\left[t_{0}, \infty\right)}\right\|=\bar{\gamma}^{-1}\left(M_{3}\right) \leq$ $\bar{\gamma}^{-1} \circ \delta_{3}\left(\left\|x\left(t_{0}\right)\right\|\right)$. Hence,

$$
\begin{aligned}
x_{\infty} & =\max \left\{\alpha_{x}\left(\left\|x\left(t_{0}\right)\right\|\right), \alpha_{u}\left(\left\|u_{\left[t_{0}, \infty\right)}\right\|\right), \alpha_{y}\left(y_{\infty}\right)\right\} \\
& \leq \max \left\{\alpha_{x}\left(\left\|x\left(t_{0}\right)\right\|\right), \alpha_{u} \circ \bar{\gamma}^{-1} \circ \delta_{3}\left(\left\|x\left(t_{0}\right)\right\|\right), \alpha_{y} \circ \delta_{3}\left(\left\|x\left(t_{0}\right)\right\|\right)\right\} \leq \delta_{4}\left(\left\|x\left(t_{0}\right)\right\|\right)
\end{aligned}
$$

for any class $K$ function $\delta_{4}$ satisfying

$\delta_{4}(s) \geq \max \left\{\alpha_{x}(s), \alpha_{u} \circ \bar{\gamma}^{-1} \circ \delta_{3}(s), \alpha_{y} \circ \delta_{3}(s)\right\}$.

As a result, (30) gives

$\left\|y_{1}(t)\right\| \leq \max \left\{\hat{\beta}_{1}\left(\delta_{4}\left(\left\|x\left(t_{0}\right)\right\|\right), t-t_{0}\right), M_{1}\right\}$.

(ii) $\delta_{3}\left(\left\|x\left(t_{0}\right)\right\|\right)<M_{3}$ : We have $y_{\infty}=M_{3}$, then $\left\|y_{1\left[t_{0}, \infty\right)}\right\| \leq y_{\infty}=M_{3}$.

In both cases, we have obtained the following inequality:

$$
\left\|y_{1}(t)\right\| \leq \max \left\{\hat{\beta}_{1}\left(\delta_{4}\left(\left\|x\left(t_{0}\right)\right\|\right), t-t_{0}\right), M_{3}\right\} .
$$

By symmetry of $y_{1}$ and $y_{2}$, we could obtain the following inequality:

$$
\left\|y_{2}(t)\right\| \leq \max \left\{\hat{\beta}_{2}\left(\delta_{4}\left(\left\|x\left(t_{0}\right)\right\|\right), t-t_{0}\right), M_{3}\right\} .
$$

Next, we will show that the system composed of (10) and (11) is RIOS with suitable defined restrictions and gain function $\bar{\gamma}$. Combing (30) and (31) gives that

$$
\begin{aligned}
\|y(t)\| & \leq\left\|y_{1}(t)\right\|+\left\|y_{2}(t)\right\| \\
& \leq \max \left\{2 \hat{\beta}_{1}\left(x_{\infty}, t-t_{0}\right), 2 \hat{\beta}_{2}\left(x_{\infty}, t-t_{0}\right), 2 M_{1}, 2 M_{2}\right\} \\
& \leq \max \left\{\beta_{3}\left(x_{\infty}, t-t_{0}\right), M_{3}\right\}
\end{aligned}
$$

for $\beta_{3}(s, t)=\max \left\{2 \hat{\beta}_{1}(s, t), 2 \hat{\beta}_{2}(s, t)\right\}$.

Toward this end, consider the following two cases of $y_{\infty}$ in (25).

(i) $\delta_{3}\left(\left\|x\left(t_{0}\right)\right\|\right) \geq M_{3}$ : We have $x_{\infty} \leq \delta_{4}\left(\left\|x\left(t_{0}\right)\right\|\right)$. As a result, (34) gives that

$$
\|y(t)\| \leq \max \left\{\beta_{3}\left(\delta_{4}\left(\left\|x\left(t_{0}\right)\right\|\right), t-t_{0}\right), M_{3}\right\} .
$$

(ii) $\delta_{3}\left(\left\|x\left(t_{0}\right)\right\|\right)<M_{3}$ : We have $y_{\infty}=M_{3}$, then $\|y(t)\| \leq M_{3}$.

In both cases, we have obtained the following inequality:

$$
\begin{aligned}
\|y(t)\| & \leq \max \left\{\beta_{3}\left(\delta_{4}\left(\left\|x\left(t_{0}\right)\right\|\right), t-t_{0}\right), M_{3}\right\} \\
& =\max \left\{\bar{\beta}\left(\left\|x\left(t_{0}\right)\right\|, t-t_{0}\right), \bar{\gamma}\left(\left\|u_{\left[t_{0}, \infty\right)}\right\|\right)\right\}
\end{aligned}
$$


where,

$$
\bar{\beta}(s, t)=\max \left\{2 \hat{\beta}_{1}\left(\delta_{4}(s), t\right), 2 \hat{\beta}_{2}\left(\delta_{4}(s), t\right)\right\} .
$$

Since the solution $y(t)$ depends only $u(\tau)$ on $t_{0} \leq \tau \leq t$, the supremum on the right hand side of (36) can be taken over $\left[t_{0}, t\right]$, which yields

$$
\|y(t)\| \leq \max \left\{\bar{\beta}\left(\left\|x\left(t_{0}\right)\right\|, t-t_{0}\right), \bar{\gamma}\left(\left\|u_{\left[t_{0}, t\right]}\right\|\right)\right\} .
$$

Hence, the system composed of (10) and (11) is RIOS with restrictions $\tilde{X}_{1} \times \tilde{X}_{2}$, $\tilde{\Delta}_{1}$ and $\tilde{\Delta}_{2}$ on $x\left(t_{0}\right), u_{1}$ and $u_{2}$ respectively, viewing $x=\operatorname{col}\left(x_{1}, x_{2}\right)$ as state, $y=$ $\operatorname{col}\left(y_{1}, y_{2}\right)$ as output and $u=\operatorname{col}\left(u_{1}, u_{2}\right)$ as input.

Step3: We will show that the system composed of (10) and (11) is RISS with restrictions $\tilde{X}_{1} \times \tilde{X}_{2}, \tilde{\Delta}_{1}$ and $\tilde{\Delta}_{2}$ on $x\left(t_{0}\right), u_{1}$ and $u_{2}$ respectively, viewing $x=$ $\operatorname{col}\left(x_{1}, x_{2}\right)$ as state, $y=\operatorname{col}\left(y_{1}, y_{2}\right)$ as output and $u=\operatorname{col}\left(u_{1}, u_{2}\right)$ as input.

Substituting (21) into (14) gives that

$\left\|x_{2}(t)\right\| \leq \max \left\{\beta_{2}\left(\left\|x_{2}\left(t_{0}\right)\right\|, t-t_{0}\right), \gamma_{2} \circ \bar{\beta}_{1}\left(\left\|x_{1}\left(t_{0}\right)\right\|, 0\right), \gamma_{2} \circ \bar{\gamma}_{1} \circ \bar{\beta}_{2}\left(\left\|x_{2}\left(t_{0}\right)\right\|, 0\right)\right.$,

$$
\left.\gamma_{2} \circ \bar{\gamma}_{1} \circ \bar{\gamma}_{2}^{u}\left(\left\|u_{2\left[t_{0}, t\right]}\right\|\right), \gamma_{2} \circ \bar{\gamma}_{1}^{u}\left(\left\|u_{1\left[t_{0}, t\right]}\right\|\right), \gamma_{2}^{u}\left(\left\|u_{2\left[t_{0}, t\right]}\right\|\right)\right\} \text {. }
$$

By symmetry of $x_{1}$ and $x_{2}$, it holds that

$$
\left\|x_{1}(t)\right\| \leq \max \left\{\beta_{1}\left(\left\|x_{1}\left(t_{0}\right)\right\|, t-t_{0}\right), \gamma_{1} \circ \bar{\beta}_{2}\left(\left\|x_{2}\left(t_{0}\right)\right\|, 0\right), \gamma_{1} \circ \bar{\gamma}_{2} \circ \bar{\beta}_{1}\left(\left\|x_{1}\left(t_{0}\right)\right\|, 0\right),\right.
$$

$$
\left.\gamma_{1} \circ \bar{\gamma}_{2} \circ \bar{\gamma}_{1}^{u}\left(\left\|u_{1\left[t_{0}, t\right]}\right\|\right), \gamma_{1} \circ \bar{\gamma}_{2}^{u}\left(\left\|u_{2\left[t_{0}, t\right]}\right\|\right), \gamma_{1}^{u}\left(\left\|u_{1\left[t_{0}, t\right]}\right\|\right)\right\} .
$$

Combing (37) and (38) gives that

$$
\|x(t)\| \leq\left\|x_{1}(t)\right\|+\left\|x_{2}(t)\right\| \leq \max \left\{\delta_{5}\left(\left\|x\left(t_{0}\right)\right\|\right), \tilde{\gamma}\left(\left\|u_{\left[t_{0}, \infty\right)}\right\|\right)\right\} \stackrel{\text { def }}{=} x_{\infty}^{\prime}
$$

where,

$$
\begin{aligned}
\delta_{5}(s, t)= & \max \left\{2 \beta_{1}(s, 0), 2 \gamma_{1} \circ \bar{\beta}_{2}(s, 0), 2 \gamma_{1} \circ \bar{\gamma}_{2} \circ \bar{\beta}_{1}(s, 0),\right. \\
& \left.2 \beta_{2}(s, 0), 2 \gamma_{2} \circ \bar{\beta}_{1}(s, 0), 2 \gamma_{2} \circ \bar{\gamma}_{1} \circ \bar{\beta}_{2}(s, 0)\right\} \\
\tilde{\gamma}(s)= & \max \left\{2 \gamma_{1} \circ \bar{\gamma}_{2} \circ \bar{\gamma}_{1}^{u}(s), 2 \gamma_{1} \circ \bar{\gamma}_{2}^{u}(s), 2 \gamma_{1}^{u}(s), 2 \gamma_{2} \circ \bar{\gamma}_{1} \circ \bar{\gamma}_{2}^{u}(s), 2 \gamma_{2} \circ \bar{\gamma}_{1}^{u}(s), 2 \gamma_{2}^{u}(s)\right\} .
\end{aligned}
$$

From (13), for any time $t_{1} \geq 0$, we could obtain

$\left\|x_{1}\left(t_{0}+t_{1}\right)\right\| \leq \max \left\{\beta_{1}\left(\left\|x_{1}\left(t_{0}+\frac{t_{1}}{2}\right)\right\|, \frac{t_{1}}{2}\right), \gamma_{1}\left(\left\|y_{2\left[t_{0}+\frac{t_{1}}{2}, t_{0}+t_{1}\right]}\right\|\right), \gamma_{1}^{u}\left(\left\|u_{1\left[t_{0}+\frac{t_{1}}{2}, t_{0}+t_{1}\right]}\right\|\right)\right\}$

$$
\leq \max \left\{\beta_{1}\left(x_{\infty}^{\prime}, \frac{t_{1}}{2}\right), \gamma_{1}\left(\left\|y_{2\left[t_{0}+\frac{t_{1}}{2}, t_{0}+t_{1}\right]}\right\|\right), \gamma_{1}^{u}\left(\left\|u_{1\left[t_{0}, \infty\right)}\right\|\right)\right\} .
$$

From (33), for $\tau \in\left[\frac{t_{1}}{2}, t_{1}\right]$, it holds that

$$
\begin{aligned}
\left\|y_{2}\left(t_{0}+\tau\right)\right\| & \leq \max \left\{\hat{\beta}_{2}\left(\delta_{4}\left(\left\|x\left(t_{0}+\frac{t_{1}}{4}\right)\right\|\right), \tau-\frac{t_{1}}{4}\right), \bar{\gamma}\left(\left\|u_{\left[t_{0}+\frac{t_{1}}{4}, t_{0}+\tau\right]}\right\|\right)\right\} \\
& \leq \max \left\{\hat{\beta}_{2}\left(\delta_{4}\left(x_{\infty}^{\prime}\right), \frac{t_{1}}{4}\right), \bar{\gamma}\left(\left\|u_{\left[t_{0}, \infty\right)}\right\|\right)\right\} .
\end{aligned}
$$


Substituting (41) into (40) gives

$$
\begin{aligned}
\left\|x_{1}\left(t_{0}+t_{1}\right)\right\| & \leq \max \left\{\beta_{1}\left(x_{\infty}^{\prime}, \frac{t_{1}}{2}\right), \gamma_{1} \circ \hat{\beta}_{2}\left(\delta_{4}\left(x_{\infty}^{\prime}\right), \frac{t_{1}}{4}\right), \gamma_{1} \circ \bar{\gamma}\left(\left\|u_{\left[t_{0}, \infty\right)}\right\|\right), \gamma_{1}^{u}\left(\left\|u_{1\left[t_{0}, \infty\right)}\right\|\right)\right\} \\
(42) & \leq \max \left\{\beta_{1}^{*}\left(x_{\infty}^{\prime}, t_{1}\right), \gamma_{1}^{*}\left(\left\|u_{\left[t_{0}, \infty\right)}\right\|\right)\right\}
\end{aligned}
$$

where, $\beta_{1}^{*}(s, t)=\max \left\{\beta_{1}\left(s, \frac{t}{2}\right), \gamma_{1} \circ \hat{\beta}_{2}\left(\delta_{4}(s), \frac{t}{4}\right)\right\}, \quad \gamma_{1}^{*}(s)=\max \left\{\gamma_{1} \circ \bar{\gamma}(s), \gamma_{1}^{u}(s)\right\}$.

By symmetry of $x_{1}$ and $x_{2}$, it holds that there exist class $K L$ function $\beta_{2}^{*}$ and class $K$ function $\gamma_{2}^{*}$ such that

$$
\left\|x_{2}(t)\right\| \leq \max \left\{\beta_{2}^{*}\left(x_{\infty}^{\prime}, t-t_{0}\right), \gamma_{2}^{*}\left(\left\|u_{\left[t_{0}, \infty\right)}\right\|\right)\right\}
$$

where, $\beta_{2}^{*}(s, t)=\max \left\{\beta_{2}\left(s, \frac{t}{2}\right), \gamma_{2} \circ \hat{\beta}_{1}\left(\delta_{4}(s), \frac{t}{4}\right)\right\}, \quad \gamma_{2}^{*}(s)=\max \left\{\gamma_{2} \circ \bar{\gamma}(s), \gamma_{2}^{u}(s)\right\}$.

Combing (42) and (43) gives

$$
\begin{aligned}
\|x(t)\| & \leq\left\|x_{1}(t)\right\|+\left\|x_{2}(t)\right\| \\
& \leq \max \left\{\beta^{*}\left(x_{\infty}^{\prime}, t-t_{0}\right), \gamma^{*}\left(\left\|u_{\left[t_{0}, \infty\right)}\right\|\right)\right\}
\end{aligned}
$$

where, $\beta^{*}(s, t)=\max \left\{2 \beta_{1}^{*}(s, t), 2 \beta_{2}^{*}(s, t)\right\}$,

$\gamma^{*}(s)=\max \left\{2 \gamma_{1}^{*}(s), 2 \gamma_{2}^{*}(s)\right\}=\max \left\{4 \gamma_{1} \circ \bar{\gamma}_{1} \circ \bar{\gamma}_{2}^{u}(s), 4 \gamma_{1} \circ \bar{\gamma}_{1}^{u}(s), 4 \gamma_{1} \circ \bar{\gamma}_{2} \circ \bar{\gamma}_{1}^{u}(s), 4 \gamma_{1} \circ\right.$ $\left.\bar{\gamma}_{2}^{u}(s), 2 \gamma_{1}^{u}(s), 4 \gamma_{2} \circ \bar{\gamma}_{1} \circ \bar{\gamma}_{2}^{u}(s), 4 \gamma_{2} \circ \bar{\gamma}_{1}^{u}(s), 4 \gamma_{2} \circ \bar{\gamma}_{2} \circ \bar{\gamma}_{1}^{u}(s), 4 \gamma_{2} \circ \bar{\gamma}_{2}^{u}(s), 2 \gamma_{2}^{u}(s)\right\}$.

Toward this end, consider the following two cases of $x_{\infty}^{\prime}$ in (39).

(i) $\delta_{5}\left(\left\|x\left(t_{0}\right)\right\|\right) \geq \tilde{\gamma}\left(\left\|u_{\left[t_{0}, \infty\right)}\right\|\right)$ : We have $x_{\infty}^{\prime}=\delta_{5}\left(\left\|x\left(t_{0}\right)\right\|\right)$.

As a result, $\|x(t)\| \leq \max \left\{\beta^{*}\left(\delta_{5}\left(\left\|x\left(t_{0}\right)\right\|\right), t-t_{0}\right), \gamma^{*}\left(\left\|u_{\left[t_{0}, \infty\right)}\right\|\right)\right\}$.

(ii) $\delta_{5}\left(\left\|x\left(t_{0}\right)\right\|\right)<\tilde{\gamma}\left(\left\|u_{\left[t_{0}, \infty\right)}\right\|\right)$ : We have $x_{\infty}^{\prime}=\tilde{\gamma}\left(\left\|u_{\left[t_{0}, \infty\right)}\right\|\right)$. As a result, $\|x(t)\| \leq x_{\infty}^{\prime}=\tilde{\gamma}\left(\left\|u_{\left[t_{0}, \infty\right)}\right\|\right)$.

Since $\tilde{\gamma}(s)<\gamma^{*}(s)$ for all $s>0$, in both cases, we have obtained the following inequality

$$
\|x(t)\| \leq \max \left\{\beta\left(\left\|x\left(t_{0}\right)\right\|, t-t_{0}\right), \gamma\left(\left\|u_{\left[t_{0}, \infty\right)}\right\|\right)\right\}
$$

where, $\beta(s, t)=\beta^{*}\left(\delta_{5}(s), t\right), \quad \gamma(s)=\gamma^{*}(s)$.

Since the solution $x(t)$ depends only on $u(\tau)$ on $t_{0} \leq \tau \leq t$, the supremum on the right hand side of (45) can be taken over $\left[t_{0}, t\right]$, which yields

$$
\|x(t)\| \leq \max \left\{\beta\left(\left\|x\left(t_{0}\right)\right\|, t-t_{0}\right), \gamma\left(\left\|u_{\left[t_{0}, t\right]}\right\|\right)\right\} .
$$

Hence, the system composed of (10) and (11) is RISS with restrictions $\tilde{X}_{1} \times \tilde{X}_{2}$, $\tilde{\Delta}_{1}$ and $\tilde{\Delta}_{2}$ on $x\left(t_{0}\right), u_{1}$ and $u_{2}$ respectively, viewing $x=\operatorname{col}\left(x_{1}, x_{2}\right)$ as state, $y=$ $\operatorname{col}\left(y_{1}, y_{2}\right)$ as output and $u=\operatorname{col}\left(u_{1}, u_{2}\right)$ as input. This completes the proof. 
REMARK 2.2. Theorem 2.2 can be viewed as an extension of Theorem B.3.2 [6] which handles the time-invariant nonlinear systems to time-varying nonlinear systems. Nevertheless, the proof of these two theorems are quite different. The proof of Theorem B.3.2 [6] is based on the separation property for time-invariant systems. However, as mentioned in Remark 1.1, the separation property does not hold for the time-varying systems. As a result, we have adopted the technique of [2] and [3] to prove steps 2 and 3 of Theorem 2.2.

3. Semi-Uniform ISS Small Gain Theorem with Restrictions for Uncertain Nonlinear Time-varying Systems. In many cases, it is easier and sufficient to ascertain the semi-uniform ISS property than the ISS property for a time-varying nonlinear system. Therefore, it is interesting to infer the semi-uniform ISS property of a feedback connected system assuming each subsystem has the semi-uniform ISS property.

Theorem 3.1. Under Assumption 2.2, assume that subsystem (10) is semiuniformly RISS and RALS with restrictions $X_{1}, \Delta_{1}$ and $\Delta_{1}^{u}$ on $x_{1}\left(t_{0}\right), v_{1}$ and $u_{1}$ respectively, i.e., there exist class $K$ functions $\gamma_{1}^{0}, \gamma_{1}, \gamma_{1}^{u}, \bar{\gamma}_{1}^{0}, \bar{\gamma}_{1}$ and $\bar{\gamma}_{1}^{u}$, independent of $d(t)$, such that, for any $x_{1}\left(t_{0}\right) \in X_{1}, v_{1}(t) \in L_{\infty}^{q_{1}}$ satisfying $\left\|v_{1\left[t_{0}, \infty\right)}\right\|<\Delta_{1}$, $u_{1}(t) \in L_{\infty}^{m_{1}}$ satisfying $\left\|u_{1\left[t_{0}, \infty\right)}\right\|<\Delta_{1}^{u}$, the solution and output of (10) exist and satisfy, for all $t \geq t_{0}$,

$$
\begin{aligned}
\left\|x_{1}(t)\right\| & \leq \max \left\{\gamma_{1}^{0}\left(\left\|x_{1}\left(t_{0}\right)\right\|\right), \gamma_{1}\left(\left\|v_{1\left[t_{0}, \infty\right)}\right\|\right), \gamma_{1}^{u}\left(\left\|u_{1\left[t_{0}, \infty\right)}\right\|\right)\right\} \\
\left\|x_{1}\right\|_{a} & \leq \max \left\{\gamma_{1}\left(\left\|v_{1}\right\|_{a}\right), \gamma_{1}^{u}\left(\left\|u_{1}\right\|_{a}\right)\right\} \\
\left\|y_{1}(t)\right\| & \leq \max \left\{\bar{\gamma}_{1}^{0}\left(\left\|x_{1}\left(t_{0}\right)\right\|\right), \bar{\gamma}_{1}\left(\left\|v_{1\left[t_{0}, \infty\right)}\right\|\right), \bar{\gamma}_{1}^{u}\left(\left\|u_{1\left[t_{0}, \infty\right)}\right\|\right)\right\} \\
\left\|y_{1}\right\|_{a} & \leq \max \left\{\bar{\gamma}_{1}\left(\left\|v_{1}\right\|_{a}\right), \bar{\gamma}_{1}^{u}\left(\left\|u_{1}\right\|_{a}\right)\right\}
\end{aligned}
$$

Also assume that subsystem (11) is semi-uniformly RISS and RALS with restrictions $X_{2}, \Delta_{2}$ and $\Delta_{2}^{u}$ on $x_{2}\left(t_{0}\right), v_{2}$ and $u_{2}$ respectively, i.e., there exist class $K$ functions $\gamma_{2}^{0}, \gamma_{2}, \gamma_{2}^{u}, \bar{\gamma}_{2}^{0}, \bar{\gamma}_{2}$ and $\bar{\gamma}_{2}^{u}$, independent of $d(t)$, such that, for any $x_{2}\left(t_{0}\right) \in X_{2}$, $v_{2}(t) \in L_{\infty}^{q_{2}}$ satisfying $\left\|v_{2\left[t_{0}, \infty\right)}\right\|<\Delta_{2}, u_{2}(t) \in L_{\infty}^{m_{2}}$ satisfying $\left\|u_{2\left[t_{0}, \infty\right)}\right\|<\Delta_{2}^{u}$, the solution and output of (11) exist and satisfy, for all $t \geq t_{0}$,

$$
\begin{aligned}
\left\|x_{2}(t)\right\| & \leq \max \left\{\gamma_{2}^{0}\left(\left\|x_{2}\left(t_{0}\right)\right\|\right), \gamma_{2}\left(\left\|v_{2\left[t_{0}, \infty\right)}\right\|\right), \gamma_{2}^{u}\left(\left\|u_{2\left[t_{0}, \infty\right)}\right\|\right)\right\} \\
\left\|x_{2}\right\|_{a} & \leq \max \left\{\gamma_{2}\left(\left\|v_{2}\right\|_{a}\right), \gamma_{2}^{u}\left(\left\|u_{2}\right\|_{a}\right)\right\} \\
\left\|y_{2}(t)\right\| & \leq \max \left\{\bar{\gamma}_{2}^{0}\left(\left\|x_{2}\left(t_{0}\right)\right\|\right), \bar{\gamma}_{2}\left(\left\|v_{2\left[t_{0}, \infty\right)}\right\|\right), \bar{\gamma}_{2}^{u}\left(\left\|u_{2\left[t_{0}, \infty\right)}\right\|\right)\right\} \\
\left\|y_{2}\right\|_{a} & \leq \max \left\{\bar{\gamma}_{2}\left(\left\|v_{2}\right\|_{a}\right), \bar{\gamma}_{2}^{u}\left(\left\|u_{2}\right\|_{a}\right)\right\} .
\end{aligned}
$$

Suppose that the small gain condition

$$
\bar{\gamma}_{1} \circ \bar{\gamma}_{2}(r)<r, \quad r>0
$$


holds. Then the system composed of (10) and (11) is semi-uniformly RISS and RALS with restrictions $\tilde{X}_{1} \times \tilde{X}_{2}, \tilde{\Delta}_{1}$ and $\tilde{\Delta}_{2}$ on $x\left(t_{0}\right), u_{1}$ and $u_{2}$ respectively, viewing $x$ $=\operatorname{col}\left(x_{1}, x_{2}\right)$ as state, $y=\operatorname{col}\left(y_{1}, y_{2}\right)$ as output and $u=\operatorname{col}\left(u_{1}, u_{2}\right)$ as input, i.e., there exist class $K$ functions $\gamma^{0}, \gamma^{u}, \bar{\gamma}^{0}$ and $\bar{\gamma}^{u}$, independent of $d(t)$, such that, for any initial state $x\left(t_{0}\right) \in \tilde{X}_{1} \times \tilde{X}_{2}$, and any input functions $u_{1}(t) \in L_{\infty}^{m_{1}}$ satisfying $\left\|u_{1\left[t_{0}, \infty\right)}\right\|<\tilde{\Delta}_{1}$ and $u_{2}(t) \in L_{\infty}^{m_{2}}$ satisfying $\left\|u_{2\left[t_{0}, \infty\right)}\right\|<\tilde{\Delta}_{2}$, the solution and output of (10) and (11) exist and satisfy, for all $t \geq t_{0}$,

$$
\begin{aligned}
\|x(t)\| \leq \max \left\{\gamma^{0}\left(\left\|x\left(t_{0}\right)\right\|\right), \gamma^{u}\left(\left\|u_{\left[t_{0}, \infty\right)}\right\|\right)\right\}, & \|x\|_{a} \leq \gamma^{u}\left(\|u\|_{a}\right) \\
\|y(t)\| \leq \max \left\{\bar{\gamma}^{0}\left(\left\|x\left(t_{0}\right)\right\|\right), \bar{\gamma}^{u}\left(\left\|u_{\left[t_{0}, \infty\right)}\right\|\right)\right\}, & \|y\|_{a} \leq \bar{\gamma}^{u}\left(\|u\|_{a}\right) .
\end{aligned}
$$

where,

$$
\begin{aligned}
\gamma^{0}(s)= & \max \left\{2 \gamma_{1}^{0}(s), 2 \gamma_{1} \circ \bar{\gamma}_{2}^{0}(s), 2 \gamma_{1} \circ \bar{\gamma}_{2} \circ \bar{\gamma}_{1}^{0}(s), 2 \gamma_{2}^{0}(s), 2 \gamma_{2} \circ \bar{\gamma}_{1}^{0}(s), 2 \gamma_{2} \circ \bar{\gamma}_{1} \circ \bar{\gamma}_{2}^{0}(s)\right\} \\
\gamma^{u}(s)= & \max \left\{2 \gamma_{1} \circ \bar{\gamma}_{2} \circ \bar{\gamma}_{1}^{u}(s), 2 \gamma_{1} \circ \bar{\gamma}_{2}^{u}(s), 2 \gamma_{1}^{u}(s)\right. \\
& \left.2 \gamma_{2} \circ \bar{\gamma}_{1} \circ \bar{\gamma}_{2}^{u}(s), 2 \gamma_{2} \circ \bar{\gamma}_{1}^{u}(s), 2 \gamma_{2}^{u}(s)\right\} \\
\bar{\gamma}^{0}(s)= & \max \left\{2 \bar{\gamma}_{1}^{0}(s), 2 \bar{\gamma}_{1} \circ \bar{\gamma}_{2}^{0}(s), 2 \bar{\gamma}_{2}^{0}(s), 2 \bar{\gamma}_{2} \circ \bar{\gamma}_{1}^{0}(s)\right\} \\
\bar{\gamma}^{u}(s)= & \max \left\{2 \bar{\gamma}_{1} \circ \bar{\gamma}_{2}^{u}(s), 2 \bar{\gamma}_{1}^{u}(s), 2 \bar{\gamma}_{2} \circ \bar{\gamma}_{1}^{u}(s), 2 \bar{\gamma}_{2}^{u}(s)\right\}
\end{aligned}
$$

and,

(i) If $\Delta_{1}, \Delta_{2}$ are finite,

$\tilde{X}_{1}=\left\{x_{1} \in X_{1}: \bar{\gamma}_{1}^{0}\left(\left\|x_{1}\right\|\right)<\Delta_{2}, \bar{\gamma}_{2} \circ \bar{\gamma}_{1}^{0}\left(\left\|x_{1}\right\|\right)<\Delta_{1}\right\}$,

and

$\tilde{X}_{2}=\left\{x_{2} \in X_{2}: \bar{\gamma}_{2}^{0}\left(\left\|x_{2}\right\|\right)<\Delta_{1}, \bar{\gamma}_{1} \circ \bar{\gamma}_{2}^{0}\left(\left\|x_{2}\right\|\right)<\Delta_{2}\right\}$.

$\tilde{\Delta}_{1} \leq \Delta_{1}^{u}, \tilde{\Delta}_{2} \leq \Delta_{2}^{u}$

$s \in\left[0, \tilde{\Delta}_{1}\right) \Longrightarrow \bar{\gamma}_{2} \circ \bar{\gamma}_{1}^{u}(s)<\Delta_{1}, \bar{\gamma}_{1}^{u}(s)<\Delta_{2}$

and

$s \in\left[0, \tilde{\Delta}_{2}\right) \Longrightarrow \bar{\gamma}_{1} \circ \bar{\gamma}_{2}^{u}(s)<\Delta_{2}, \bar{\gamma}_{2}^{u}(s)<\Delta_{1}$.

(ii) If $\Delta_{1}, \Delta_{2}$ are infinite,

$\tilde{X}_{1}=X_{1}, \tilde{X}_{2}=X_{2}$

and

$\tilde{\Delta}_{1} \leq \Delta_{1}^{u}, \tilde{\Delta}_{2} \leq \Delta_{2}^{u}$.

Proof. The proof can be obtained by using the same technique used in the proof of Theorem 1 of [20] and is thus omitted.

Corollary 3.1. Consider the interconnection of the following two systems

$$
\begin{aligned}
& \dot{x}_{1}=f_{1}\left(x_{1}, u_{1}, d, t\right), \quad y_{1}=x_{1} \\
& \dot{x}_{2}=f_{2}\left(x_{2}, v_{2}, u_{2}, d, t\right), \quad y_{2}=x_{2}
\end{aligned}
$$


subject to the interconnection constraint:

$$
v_{2}=y_{1}
$$

where, the notations are the same as those in Theorem 3.1.

Assume that subsystem (57) is semi-uniformly RISS with restrictions $X_{1}$ and $\Delta_{1}^{u}$ on $x_{1}\left(t_{0}\right)$ and $u_{1}$ respectively, i.e., there exist class $K$ functions $\gamma_{1}^{0}, \gamma_{1} \equiv 0$ and $\gamma_{1}^{u}$, independent of $d(t)$, such that, for any $x_{1}\left(t_{0}\right) \in X_{1}, u_{1}(t) \in L_{\infty}^{m_{1}}$ satisfying $\left\|u_{1\left[t_{0}, \infty\right)}\right\|<\Delta_{1}^{u}$, the solution and output of (57) exist and, for all $t \geq t_{0}$, (46) and (47) holds.

Also assume that subsystem (58) is semi-uniformly RISS with restrictions $X_{2}, \Delta_{2}$ and $\Delta_{2}^{u}$ on $x_{2}\left(t_{0}\right), v_{2}$ and $u_{2}$ respectively, i.e., there exist class $K$ functions $\gamma_{2}^{0}, \gamma_{2}$ and $\gamma_{2}^{u}$, independent of $d(t)$, such that, for any $x_{2}\left(t_{0}\right) \in X_{2}, v_{2}(t) \in L_{\infty}^{q_{2}}$ satisfying $\left\|v_{2\left[t_{0}, \infty\right)}\right\|<\Delta_{2}, u_{2}(t) \in L_{\infty}^{m_{2}}$ satisfying $\left\|u_{2\left[t_{0}, \infty\right)}\right\|<\Delta_{2}^{u}$, the solution and output of (58) exist and, for all $t \geq t_{0},(50)$ and (51) hold.

Then the system composed of (57) and (58) is semi-uniformly RISS with restrictions $\tilde{X}_{1} \times \tilde{X}_{2}, \tilde{\Delta}_{1}$ and $\tilde{\Delta}_{2}$ on $x\left(t_{0}\right), u_{1}$ and $u_{2}$ respectively, viewing $x=\operatorname{col}\left(x_{1}, x_{2}\right)$ as state, $y=\operatorname{col}\left(y_{1}, y_{2}\right)$ as output and $u=\operatorname{col}\left(u_{1}, u_{2}\right)$ as input, i.e., there exist class $K$ functions $\gamma^{0}, \gamma^{u}, \bar{\gamma}^{0}$ and $\bar{\gamma}^{u}$, independent of $d(t)$, such that, for any initial state $x\left(t_{0}\right) \in \tilde{X}_{1} \times \tilde{X}_{2}$, and any input functions $u_{1}(t) \in L_{\infty}^{m_{1}}$ satisfying $\left\|u_{1\left[t_{0}, \infty\right)}\right\|<\tilde{\Delta}_{1}$ and $u_{2}(t) \in L_{\infty}^{m_{2}}$ satisfying $\left\|u_{2\left[t_{0}, \infty\right)}\right\|<\tilde{\Delta}_{2}$, the solution and output of (57) and (58) exist and satisfy, for all $t \geq t_{0}$,

$$
\|x(t)\| \leq \max \left\{\gamma^{0}\left(\left\|x\left(t_{0}\right)\right\|\right), \gamma^{u}\left(\left\|u_{\left[t_{0}, \infty\right)}\right\|\right)\right\}, \quad\|x\|_{a} \leq \gamma^{u}\left(\|u\|_{a}\right)
$$

where,

$$
\begin{aligned}
& \gamma^{0}(s)=\max \left\{2 \gamma_{1}^{0}(s), 2 \gamma_{2}^{0}(s), 2 \gamma_{2} \circ \bar{\gamma}_{1}^{0}(s)\right\} \\
& \gamma^{u}(s)=\max \left\{2 \gamma_{1}^{u}(s), 2 \gamma_{2}^{u}(s), 2 \gamma_{2} \circ \bar{\gamma}_{1}^{u}(s)\right\}
\end{aligned}
$$

and,

$\tilde{X}_{1}=\left\{x_{1} \in X_{1}: \bar{\gamma}_{1}^{0}\left(\left\|x_{1}\right\|\right)<\Delta_{2}\right\}, \quad \tilde{X}_{2}=X_{2}$.

$\tilde{\Delta}_{1} \leq \Delta_{1}^{u}, \tilde{\Delta}_{2} \leq \Delta_{2}^{u}$

$s \in\left[0, \tilde{\Delta}_{1}\right) \Longrightarrow \bar{\gamma}_{1}^{u}(s)<\Delta_{2}$.

\section{Asymptotic Small Gain Theorem with Restrictions for Uncertain}

Nonlinear Time-varying Systems. In this section, we will present the asymptotic small gain theorem with restrictions for uncertain nonlinear time-varying systems. The proof is quite similar to that of Theorem 2 of [20] and is thus skipped.

Theorem 4.1. Under Assumption 2.2, assume that both subsystems (10) and (11) are RAG and o-RAG with restrictions $X_{i}, \Delta_{i}$ and $\Delta_{i}^{u}$ on $x_{i}\left(t_{0}\right), v_{i}$ and $u_{i}$, 
$i=1,2$, respectively, i.e., for $i=1,2$, there exist class $K$ functions $\gamma_{i}, \gamma_{i}^{u}, \bar{\gamma}_{i}$ and $\bar{\gamma}_{i}^{u}$, independent of $d(t)$, such that, for any $x_{i}\left(t_{0}\right) \in X_{i}, v_{i}(t) \in L_{\infty}^{q_{i}}$ satisfying $\left\|v_{i}\right\|_{a} \leq \Delta_{i}$, $u_{i}(t) \in L_{\infty}^{m_{i}}$ satisfying $\left\|u_{i}\right\|_{a} \leq \Delta_{i}^{u}$, the solutions of (10) and (11) exist and satisfy, for all $t \geq t_{0}$,

$$
\begin{aligned}
\left\|x_{i}\right\|_{a} & \leq \max \left\{\gamma_{i}\left(\left\|v_{i}\right\|_{a}\right), \gamma_{i}^{u}\left(\left\|u_{i}\right\|_{a}\right)\right\} \\
\left\|y_{i}\right\|_{a} & \leq \max \left\{\bar{\gamma}_{i}\left(\left\|v_{i}\right\|_{a}\right), \bar{\gamma}_{i}^{u}\left(\left\|u_{i}\right\|_{a}\right)\right\}
\end{aligned}
$$

Suppose

Assumption 4.1. For all initial state in $X_{1} \times X_{2}$ and all piecewise continuous $u_{1}, u_{2}, d$ which are bounded on $\left[t_{0}, \infty\right)$, the solution of (10) and (11) with connection (12) is defined for all $t \geq t_{0}$;

Assumption 4.2. $\Delta_{1}=\infty$;

Assumption 4.3. $\bar{\gamma}_{1}(\infty)<\infty$ and $\bar{\gamma}_{1}(\infty) \leq \Delta_{2}$;

Assumption 4.4. The small gain condition

$$
\bar{\gamma}_{1} \circ \bar{\gamma}_{2}(r)<r, \quad r>0
$$

holds.

Then, under connection (12), the system composed of (10) and (11) is RAG and o-RAG with restrictions $X_{1} \times X_{2}, \tilde{\Delta}_{1}$ and $\tilde{\Delta}_{2}$ on $x\left(t_{0}\right), u_{1}$ and $u_{2}$ respectively, viewing $x=\operatorname{col}\left(x_{1}, x_{2}\right)$ as state, $y=\operatorname{col}\left(y_{1}, y_{2}\right)$ as output and $u=\operatorname{col}\left(u_{1}, u_{2}\right)$ as input, i.e., there exist class $K$ functions $\gamma^{u}$ and $\bar{\gamma}^{u}$, independent of $d(t)$, such that, for any initial state $x\left(t_{0}\right) \in X_{1} \times X_{2}$, and any input functions $u_{1}(t) \in L_{\infty}^{m_{1}}$ satisfying $\left\|u_{1}\right\|_{a} \leq \tilde{\Delta}_{1}$ and $u_{2}(t) \in L_{\infty}^{m_{2}}$ satisfying $\left\|u_{2}\right\|_{a} \leq \tilde{\Delta}_{2}$, the solution of (10) and (11) with connection (12) exists and satisfies, for all $t \geq t_{0}$,

$$
\begin{gathered}
\|x\|_{a} \leq \gamma^{u}\left(\|u\|_{a}\right), \\
\|y\|_{a} \leq \bar{\gamma}^{u}\left(\|u\|_{a}\right) .
\end{gathered}
$$

where,

$\gamma^{u}(s)=\max \left\{2 \gamma_{1} \circ \bar{\gamma}_{2} \circ \bar{\gamma}_{1}^{u}(s), 2 \gamma_{1} \circ \bar{\gamma}_{2}^{u}(s), 2 \gamma_{1}^{u}(s), 2 \gamma_{2} \circ \bar{\gamma}_{1} \circ \bar{\gamma}_{2}^{u}(s), 2 \gamma_{2} \circ \bar{\gamma}_{1}^{u}(s), 2 \gamma_{2}^{u}(s)\right\}$,

$\bar{\gamma}^{u}(s)=\max \left\{2 \bar{\gamma}_{1} \circ \bar{\gamma}_{2}^{u}(s), 2 \bar{\gamma}_{1}^{u}(s), 2 \bar{\gamma}_{2} \circ \bar{\gamma}_{1}^{u}(s), 2 \bar{\gamma}_{2}^{u}(s)\right\}$

and $\tilde{\Delta}_{2}$ is such that $\tilde{\Delta}_{2} \leq \Delta_{2}^{u}$,

and $\tilde{\Delta}_{1}$ is such that $\tilde{\Delta}_{1} \leq \Delta_{1}^{u}$, and $\bar{\gamma}_{1}^{u}\left(\tilde{\Delta}_{1}\right) \leq \Delta_{2}$.

Corollary 4.1. Consider the interconnection $v_{2}=y_{1}$ of the following two systems

$$
\begin{aligned}
& \dot{x}_{1}=f_{1}\left(x_{1}, u_{1}, d, t\right), \quad y_{1}=x_{1} \\
& \dot{x}_{2}=f_{2}\left(x_{2}, v_{2}, u_{2}, d, t\right), \quad y_{2}=x_{2}
\end{aligned}
$$


where, the notations are the same as those in Theorem 4.1. Suppose:

Assumption 4.5. For all initial state in $X_{1} \times X_{2}$ and all piecewise continuous $u_{1}, u_{2}, d$ which are bounded on $\left[t_{0}, \infty\right)$, the solution of (64) and (65) with connection $v_{2}=y_{1}$ is defined for all $t \geq t_{0}$;

Assumption 4.6. Subsystem (64) is RAG with restrictions $X_{1}$ and $\Delta_{1}^{u}$ on $x_{1}\left(t_{0}\right)$ and $u_{1}$ respectively;

Assumption 4.7. Subsystem (65) is RAG with restrictions $X_{2}$ and $\Delta_{2}^{u}$ on $x_{2}\left(t_{0}\right)$ and $u_{2}$ respectively.

Then system (64) and (65) with connection $v_{2}=y_{1}$ is RAG with restrictions $X_{1} \times X_{2}, \Delta_{1}^{u}, \Delta_{2}^{u}$ on $\left(x_{1}\left(t_{0}\right), x_{2}\left(t_{0}\right)\right)$ and $u_{1}^{u}, u_{2}^{u}$ respectively, i.e., there exist class $K$ function $\gamma^{u}$, independent of $d(t)$, such that, for any initial state $x\left(t_{0}\right) \in X_{1} \times X_{2}$, and any input functions $u_{1}(t) \in L_{\infty}^{m_{1}}$ satisfying $\left\|u_{1}\right\|_{a} \leq \Delta_{1}^{u}$ and $u_{2}(t) \in L_{\infty}^{m_{2}}$ satisfying $\left\|u_{2}\right\|_{a} \leq \Delta_{2}^{u}$, the solution of (64) and (65) with connection $v_{2}=y_{1}$ exists and satisfies, for all $t \geq t_{0}$,

$$
\|x\|_{a} \leq \gamma^{u}\left(\|u\|_{a}\right)
$$

where $\gamma^{u}(s)=\max \left\{2 \gamma_{2} \circ \gamma_{1}^{u}(s), 2 \gamma_{1}^{u}(s), 2 \gamma_{2}^{u}(s)\right\}$ and all the gain functions are defined the same way as those in Theorem 4.1.

The following corollary is similar to Proposition 1 in [1].

Corollary 4.2. Consider the interconnections

$$
v_{21}=y_{11}, \quad v_{22}=y_{12}, \quad v_{1}=y_{2}
$$

of the following two systems

$$
\begin{aligned}
\Sigma_{1}: \dot{x}_{1} & =f_{1}\left(x_{1}, v_{1}, d, t\right) \\
y_{11} & =h_{11}\left(x_{1}, v_{1}, d, t\right), \quad y_{12}=h_{12}\left(x_{1}, v_{1}, d, t\right) \\
\Sigma_{2}: \dot{x}_{2} & =f_{2}\left(x_{2}, v_{21}, v_{22}, u_{2}, d, t\right), \\
y_{2} & =h_{2}\left(x_{2}, v_{21}, v_{22}, u_{2}, d, t\right) .
\end{aligned}
$$

Suppose:

Assumption 4.8. For all initial state in $X_{1} \times X_{2}$ and all piecewise continuous $u_{2}, d$ which are bounded on $\left[t_{0}, \infty\right)$, the solution of $\Sigma_{1}$ and $\Sigma_{2}$ with connection (66) is defined for all $t \geq t_{0}$;

Assumption 4.9. Subsystem $\Sigma_{2}$ is RAG and o-RAG with restriction $\Delta_{22}$ on the input $v_{22}$, i.e., there exist class $K$ functions $\gamma_{21}, \gamma_{22}, \gamma_{2}^{u}, \bar{\gamma}_{21}, \bar{\gamma}_{22}$ and $\bar{\gamma}_{2}^{u}$, independent of $d(t)$, such that for any initial state $x_{2}\left(t_{0}\right) \in \Re^{n_{2}}$ and any input $v_{22}(t)$ satisfying $\left\|v_{22}\right\|_{a} \leq \Delta_{22}$, the solution of $\Sigma_{2}$ exists and satisfies, for all $t \geq t_{0}$,

$$
\begin{aligned}
\left\|x_{2}\right\|_{a} & \leq \max \left\{\gamma_{21}\left(\left\|v_{21}\right\|_{a}\right), \gamma_{22}\left(\left\|v_{22}\right\|_{a}\right), \gamma_{2}^{u}\left(\left\|u_{2}\right\|_{a}\right)\right\} \\
\left\|y_{2}\right\|_{a} & \leq \max \left\{\bar{\gamma}_{21}\left(\left\|v_{21}\right\|_{a}\right), \bar{\gamma}_{22}\left(\left\|v_{22}\right\|_{a}\right), \bar{\gamma}_{2}^{u}\left(\left\|u_{2}\right\|_{a}\right)\right\} .
\end{aligned}
$$


Assumption 4.10. Subsystem $\Sigma_{1}$ is RAG and o-RAG without restriction, i.e., there exist class $K$ functions $\gamma_{1}, \bar{\gamma}_{11}$ and $\bar{\gamma}_{12}$, independent of $d(t)$, such that for any initial state $x_{1}\left(t_{0}\right) \in \Re^{n_{1}}$ and any input $v_{1}(t)$, the solution of $\Sigma_{1}$ exists and satisfies, for all $t \geq t_{0}$,

$$
\begin{aligned}
\left\|x_{1}\right\|_{a} & \leq \gamma_{1}\left(\left\|v_{1}\right\|_{a}\right), \\
\left\|y_{11}\right\|_{a} & \leq \bar{\gamma}_{11}\left(\left\|v_{1}\right\|_{a}\right), \\
\left\|y_{12}\right\|_{a} & \leq \bar{\gamma}_{12}\left(\left\|v_{1}\right\|_{a}\right) .
\end{aligned}
$$

Assumption 4.11. $\bar{\gamma}_{11}(\infty)<\infty, \bar{\gamma}_{12}(\infty)<\infty$ and $\bar{\gamma}_{12}(\infty) \leq \Delta_{22}$

Assumption 4.12. The small gain conditions hold

$$
\bar{\gamma}_{11} \circ \bar{\gamma}_{21}(r)<r, \quad \bar{\gamma}_{12} \circ \bar{\gamma}_{22}(r)<r, \quad r>0 .
$$

Then under the interconnection (66), the system composed of $\Sigma_{1}$ and $\Sigma_{2}$ is RAG.

\section{REFERENCES}

[1] M. Arcak, A.R. Teel And P. Kokotovic, Robust nonlinear control of feedforward systems with unmodeled dynamics, Automatica, 37(2001), pp. 265-272.

[2] Z. Chen And J. HuAng, A simplified small gain theorem for time-varying nonlinear systems, IEEE Transactions on Automatic Control, 50(2005), pp. 1904-1908.

[3] Z. Chen And J. Huang, Robust input-to-state stability and small gain theorem for nonlinear systems containing time-varying uncertainty, Advanced Robust and Adaptive Control Theory and Applications, Springer-Tsinghua, 2005, pp.31-40.

[4] D.J. HiLl, A generalization of the small-gain theorem for nonlinear feedback systems, Automatica, 27(1991), pp. 1043-1045.

[5] A. Isidori, Nonlinear control systems, New York: Springer-Verlag, vol. II, 1999.

[6] A. Isidori, L. Marconi, And A. Serrani, Robust autonomous guidance: An internal model approach, New York: Springer, 2003.

[7] Z. P. Jiang, A. R. Teel, And L. Praly, Small-gain theorem for ISS sysems and applications, Mathematics of Control, Signals and Systems, 7(1994), pp. 95-120.

[8] Z. P. Jiang And I. Mareels, A small-gain control method for nonliear cascaded systems with dynamic uncertainties, IEEE Transactions on Automatic Control, 42(1997), pp. 292-308.

[9] Z. P. JiAng AND Y. WANG, Small-gain theorem on input-to-output stability, Proceedings of the Third International DCDIS Conference, pp. 220-224, 2003.

[10] H. Khalil, Nonlinear systems, Prince Hall, Upper Saddle River, New Jersey, 3rd edition, 2002.

[11] Y. Lin, Lyapunov function techniques for stabilization, Ph.D. thesis, Rutgers University, 1992.

[12] Y. Lin, E. D. Sontag, AND Y. WANG, Recent results on Lyapunov theoretic techniques for nonlniear stability, Report SYCON-93-09. 
[13] Y. Lin, E.D. Sontag, AND Y. WAng, Input to state stability for parameterized families of systems, International Journal of Robust and Nonlinear Control, 5(1995), pp. 187-205.

[14] Y. LIN, Y. WANG, AND D. Z. Cheng, On nonuniform and semi-uniform input-to-state stbility for time varying systems, Proceedings of the 16th IFAC World Congress, July 2005.

[15] E. D. Sontag, Smooth stabilization implies comprime factorization, IEEE Transactions on Automatic Control, 34(1989), pp. 435-443.

[16] E. D. SontaG, On the input-to-state stability property, Journal of Control, 1(1995), pp. 24-36.

[17] E. D. Sontag And Y. WAng, On the characteristics of the input-to-state stability property, Systems and Control Letters, 24(1995), pp. 351-359.

[18] E. D. Sontag And Y. WAng, New characterization of input-to-state stability, IEEE Transactions on Automatic Control, 41(1996), pp. 1283-1294.

[19] E. D. Sontag And Y. Wang, Notions of input to output stability, Systems and Control Letters, 38(1999), pp. 351-359.

[20] A. R. TEEL, A nonlinear small gain theorem for the analysis of control systems with saturation, IEEE Transactions on Automatic Control, 41(1996), pp. 1256-1270. 